\title{
Synthetic Aperture Hitchhiker Imaging
}

\author{
Can Evren Yarman, Member, IEEE, and Birsen Yazıc1, Senior Member, IEEE
}

\begin{abstract}
We introduce a novel synthetic-aperture imaging method for radar systems that rely on sources of opportunity. We consider receivers that fly along arbitrary, but known, flight trajectories and develop a spatio-temporal correlation-based filtered-backprojection-type image reconstruction method. The method involves first correlating the measurements from two different receiver locations. This leads to a forward model where the radiance of the target scene is projected onto the intersection of certain hyperboloids with the surface topography. We next use microlocal techniques to develop a filtered-backprojection-type inversion method to recover the scene radiance. The method is applicable to both stationary and mobile, and cooperative and noncooperative sources of opportunity. Additionally, it is applicable to nonideal imaging scenarios such as those involving arbitrary flight trajectories, and has the desirable property of preserving the visible edges of the scene radiance. We present an analysis of the computational complexity of the image reconstruction method and demonstrate its performance in numerical simulations for single and multiple transmitters of opportunity.
\end{abstract}

Index Terms-Generalized filtered-backprojection, microlocal analysis, passive imaging, radar, synthetic aperture imaging.

\section{INTRODUCTION}

A hitchhiker is a passive radar receiver that relies on sources of opportunity to perform radar tasks [1], [2]. With the rapid growth in the number of TV and radio broadcasting stations [4]-[8], mobile phone base stations [9], [10] in addition to terrestrial and space-based communication and navigation satellites [11]-[16], hitchhikers offer a viable approach to urban and rural imaging either as a stand-alone system or adjunct to active radar systems.

A synthetic-aperture radar (SAR) [17], [1] system is one that uses an antenna on a moving platform, such as an aircraft or a satellite, and which forms an effective long aperture by coherently combining views from different locations. In this paper, we consider a synthetic-aperture imaging system consisting of receivers traversing arbitrary flight trajectories that use sources of opportunity for imaging as illustrated in Fig. 1.

Manuscript received August 3, 2007; revised June 22, 2008. Current version published October 10, 2008. This work was supported by the Air Force Office of Scientific Research under the agreements FA9550-04-1-0223 and FA9550-07-1-0363. Because of this support, the U.S. Government is authorized to reproduce and distribute reprints for Governmental purposes notwithstanding any copyright notation thereon. The views and conclusions contained herein are those of the authors and should not be interpreted as necessarily representing the official policies or endorsements, either expressed or implied, of the Air Force Research Laboratory or the U.S. Government. The associate editor coordinating the review of this manuscript and approving it for publication was Prof. Peter C. Doerschuk.

C. E. Yarman is with the Houston Technology Center, WesternGeco-Schlumberger, Houston, TX 77042 USA (e-mail: yarman @ecse.rpi.edu).

B. Yazıcı is with the Electrical, Computer, and System Engineering Department, Rensselaer Polytechnic Institute, Troy, NY 12180 USA (e-mail: yazici@ecse.rpi.edu).

Digital Object Identifier 10.1109/TIP.2008.2002839
Due to its combined synthetic aperture and hitchhiking structure, we refer to the system under consideration as synthetic aperture hitchhiker (SAH). We introduce a novel spatio-temporal-correlation-based, filtered-backprojection-type image reconstruction method for SAH imaging. This method first correlates the received signals measured at different locations on the receiver flight trajectory(ies), and next applies a microlocal based filtered-backprojection technique on the correlated measurements. The method has the following practical advantages: 1) as compared to the existing passive radar detection systems [12]-[14], [5], [7], [9], [18], [8], it does not necessarily require receivers with high directivity; 2 ) it can be used in the presence of both cooperative and noncooperative sources of opportunity; 3 ) it can be used with stationary and/or mobile sources of opportunity; 4) it can be used with one or more airborne receivers; 5) it can handle nonideal imaging scenarios such as arbitrary flight trajectories or nonflat topography; 6) it has the desirable property that the visible edges in the image not only appear at the right location and right orientation but also at the right strength in the reconstructed image for the case of cooperative sources; 7) it is a noniterative, analytic image reconstruction technique which can be made computationally efficient [19].

There are two equivalent spatio-temporal correlation-based imaging approaches [20]-[26]. In the first approach, signals received from different receiver locations are backpropagated to focus at each point of interest and images obtained from each receiver pair are summed to form the final image [22], [25], [26]. In the second approach, for each pair of receivers an image is formed by first correlating the received signals from different receiver locations and then backprojecting the correlated measurements into the image domain. The final image is formed by averaging over the images obtained for each receiver pair [20], [21], [23], [24]. In both methods, the image represents the incoherent-field approximation of the target scene radiance.

To the best of our knowledge, both classes of methods consider imaging with discrete sparse apertures where the receivers and/or transmitters are static. In this paper we consider synthetic aperture imaging and present a new image reconstruction method that falls into the second type of spatio-temporal correlation imaging methods.

Our treatment combines the spatio-temporal correlation methods presented in [27], [21], [24] with the microlocal techniques [28]-[30] to develop a filtered-backprojection (FBP)-type reconstruction methods for SAH, which we refer to as correlation filtered-backprojection (C-FBP). Given multiple sparsely distributed receivers, the spatio-temporal correlation method correlates the measurements from different receivers to detect targets within the illuminated scene by means of relative change [31], [27], [32]. This process eliminates the need for knowledge about the transmitter location and waveform. The correlation process also leads to a forward model in which 
the scene radiance is projected onto the intersection $H_{i j}$ of the hyperboloid with the ground topography (see Section IV for the definition of $H_{i j}$ ). Microlocal techniques provide an approximate FBP-type inversion with the advantages outlined in (5)-(7) above. Additionally, if an exact inversion is possible, microlocal techniques often provide the exact inversion formula. Thus, the C-FBP method performs reconstruction in three steps: First, it correlates the received signals at different receiver locations; next, it filters the correlated signal; and finally, the resulting signal is backprojected along the intersection $H_{i j}$ of the illuminated surface and the hyperboloid. For ease of exposition, we present our results for the case of static sources of opportunity; however, extensions to the case of mobile sources of opportunity is straightforward. We compare our method to the backprojection method, present the analysis of the computational complexity of both methods, and compare their performances in numerical simulations.

The organization of the rest of the paper is as follows. In Section II, we introduce the synthetic aperture hitchhiker and present the forward model. In Section III, we present the spatio-temporal correlated signal model for noncooperative and cooperative sources of opportunity. In Section IV, we present the correlation-filtered-backprojection-type image reconstruction methods for both cooperative and noncooperative sources of opportunity. In Section V, we present our reconstruction algorithm and its computational complexity analysis. In Section VI, we demonstrate the performance of our method in numerical simulations for single and multiple receiver and transmit antennas for both cooperative and noncooperative sources of opportunity. Finally, we conclude our discussion in Section VII. The paper includes an appendix on the stationary phase theorem, which is repeatedly used throughout the paper, and a table of notations.

\section{TABLE OF NOTATIONS}

$\gamma_{R_{i}}(s)$
$\mathbf{x} \in \mathbb{R}^{3}$ or
$\mathbf{x}=(\boldsymbol{x}, \psi(\boldsymbol{x}))$
$\mathbf{y}$
$d_{i, \mathbf{y}}(t, s)$
$t$
$s$
$c_{0}$
$r_{i, \mathbf{y}}(s, \mathbf{x}) / c_{0}$
$G(\boldsymbol{x})$
$J_{R_{i}}$
$J_{T}$
$d_{i j}\left(t, s, s^{\prime}\right)$
$s^{\prime}$
$C_{G}\left(\boldsymbol{x}, \boldsymbol{x}^{\prime}\right)$

Flight trajectory of $i$ th receiver.

A point in space or on the surface of the earth.

Transmitter location.

Received signal at the $i$ th receiver due to transmitter at $\mathbf{y}(s)$.

Fast-time.

Slow-time.

Speed of light in free-space.

Total travel time.

Scene reflectivity.

$i$ th receiver antenna beam pattern.

Transmitter antenna beam pattern.

Spatio-temporal correlation of $d_{i}$ and $d_{j}$.

Slow-time delay.

Correlation function of $G$.
$C_{J_{T}}\left(\omega, \boldsymbol{x}, \boldsymbol{x}^{\prime}, \mathbf{y}, \mathbf{y}^{\prime}\right)$ Correlation function of $J_{T}$.

$\begin{array}{ll}R_{G}(\boldsymbol{x}) & \text { Scene radiance. } \\ R_{T}(\omega, \boldsymbol{x}, \mathbf{y}) & \text { Transmitter irradiance. } \\ r_{i j} & \text { Hitchhiker range. } \\ \tilde{R}_{T}(\omega, \boldsymbol{x}) & \text { Total average power incident upon } \boldsymbol{x} . \\ \mathcal{F} & \text { Forward modeling operator. } \\ H_{i j}\left(t, s, s^{\prime}\right) & \text { Iso-range contours. } \\ \mathcal{K} & \text { Filtered-backprojection operator. } \\ Q_{i j} & \text { Filter of } \mathcal{K} \text {. } \\ \hat{R}_{G}(\boldsymbol{z}) & \text { Reconstructed image of } R_{G}(\boldsymbol{z}) . \\ L(\boldsymbol{z}, \boldsymbol{x}) & \text { Point spread function. } \\ \delta(\boldsymbol{x}) & \text { Dirac delta function. } \\ f_{i j} & \text { Hitchhiker Doppler. } \\ F_{i j}\left(s, s^{\prime}, C\right) & \text { Iso-Doppler contours. } \\ \eta\left(\boldsymbol{x}, \boldsymbol{z}, \xi_{i j}, s^{\prime}\right) & \text { Determinant of the Jacobian. } \\ \boldsymbol{\Xi}_{i j}\left(s, s^{\prime}, \boldsymbol{z}, \boldsymbol{z}\right) & \text { See (36). } \\ \chi_{\Omega_{i j}, \boldsymbol{z}, s^{\prime}} & \text { Smooth cut-off function. } \\ \Omega_{\boldsymbol{z}} & \text { Data collection manifold. } \\ \Omega_{i j, \boldsymbol{z}, s^{\prime}} & \text { Partial data collection manifold. } \\ |\omega| & \text { Ram-Lak/Ramp filter. } \\ \hat{R}_{G_{i j}}(\boldsymbol{z}) & \text { Partial reconstructed image of } \hat{R}_{G}(\boldsymbol{z}) .\end{array}$

\section{Measurement Model}

Assume that there are $N \geq 1$ airborne antennas flying over a scene as shown in Fig. 1 . Let $\boldsymbol{\gamma}_{R_{i}}(s), s \in \mathbb{R}, i=1, \ldots, N$, be the $i$ th SAH trajectory. Let $\mathbf{x}=(\boldsymbol{x}, \psi(\boldsymbol{x})) \in \mathbb{R}^{3}$ denote the surface of the earth, where $\boldsymbol{x}=\left(x_{1}, x_{2}\right)$ and $\psi: \mathbb{R}^{2} \rightarrow \mathbb{R}$ is a known smooth function.

We assume that the electromagnetic waves propagate in free space and then scatter in a thin region at the earth's surface. Under the start-stop approximation, the single-scattering (Born) approximation of the contribution to the received signal at the $i$ th receiver, $i=1, \ldots, N$, due to a transmitter located at $\mathbf{y} \in \mathbb{R}^{3}$ can be modeled as [28]

$$
\begin{aligned}
d_{i, \mathbf{y}}(t, s) \approx \int \mathrm{e}^{-\mathrm{i} 2 \pi \omega\left(t-r_{i, \mathbf{y}}(s, \boldsymbol{x}) / c_{0}\right)} G(\boldsymbol{x}) & \\
& \times A_{R_{i}}(\omega, s, \boldsymbol{x}) A_{T}(\omega, \boldsymbol{x}, \mathbf{y}) d \omega d \boldsymbol{x}
\end{aligned}
$$

where $\mathrm{i}=\sqrt{-1}, t$ is the fast-time variable, $s$ is the slow-time variable which parameterizes the antenna trajectory, $c_{0}$ denotes the speed of light in free-space, $r_{i, \mathbf{y}}(s, \mathbf{x}) / c_{0}$, where $r_{i, \mathbf{y}}=$ $|\mathbf{y}-\mathbf{x}|+\left|\mathbf{x}-\boldsymbol{\gamma}_{R_{i}}(s)\right|$, is the total travel time, $G(\boldsymbol{x})$ denotes the scene reflectivity, and

$$
\begin{aligned}
A_{R_{i}}(\omega, s, \boldsymbol{x}) & =\sqrt{\frac{\pi}{2}} \frac{\omega J_{R_{i}}(\omega, s, \boldsymbol{x})}{\left|\gamma_{R_{i}}(s)-\mathbf{x}\right|} \\
A_{T}(\omega, \boldsymbol{x}, \mathbf{y}) & =\sqrt{\frac{\pi}{2}} \frac{\omega J_{T}(\omega, \boldsymbol{x}, \mathbf{y})}{|\mathbf{x}-\mathbf{y}|} .
\end{aligned}
$$




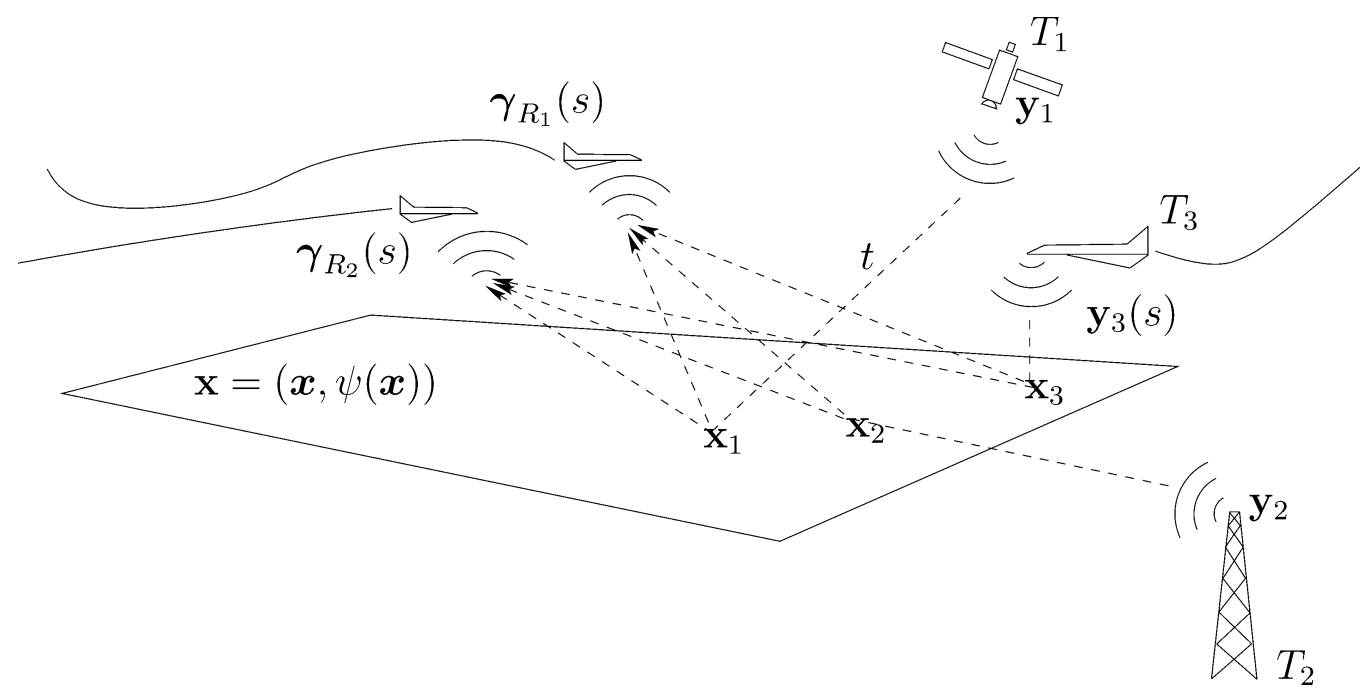

Fig. 1. Synthetic aperture hitchhiker geometry. A point on the earth's surface is denoted by $\mathbf{x}=(\boldsymbol{x}, \psi(\boldsymbol{x})) \in \mathbb{R}^{3}$, where $\boldsymbol{x} \in \mathbb{R}^{2}$ and $\psi(\boldsymbol{x}) \in \mathbb{R}$ denotes ground topography; $t$ and $s$ are the fast-time and slow-time variables used to parameterize the measurement time and flight trajectories; $\gamma_{R_{i}}(s)$ denotes the trajectory of the $i$ th receiver; $T_{i}$ denotes $i$ th transmitter, at location $\mathbf{y}_{i}(s)$. For static transmitters $\mathbf{y}_{i}(s)=\mathbf{y}_{i}$.

In (3), $J_{R_{i}}$ is the $i$ th receiver antenna beam pattern and $J_{T}$ is the transmitter antenna beam pattern located at $\mathbf{y}$ (which also includes the transmitted waveform). Note that for the current discussion we will only consider static sources of opportunity to simplify our notation. However, the model in (1) and (3) can be easily extended to mobile sources of opportunity by introducing a slow-time $(s)$ dependence in the transmitter antenna beam pattern, i.e., $J_{T}(\omega, s, \boldsymbol{x}, \mathbf{y}(s))$. The discussion in the rest of the paper applies equally well to this case.

The received signal at the $i$ th receiver is given by the superposition of (1) over all transmitters

$$
d_{i}(t, s)=\int d_{i, \mathbf{y}}(t, s) d \mathbf{y}
$$

Note that without loss of generality the integration above can be replaced with a summation for finitely many sources of opportunity.

Standard SAR image reconstruction problem involves estimation of scene reflectivity, $G$, from the measurements $d_{i}(t, s)$, for some range $\left[s_{a}, s_{b}\right]$ and $\left[0, t_{0}\right]$ of $s$ and $t$, respectively. For monostatic SAR and BISAR one approach for estimating $G$ is to perform matched filtering followed by filtered backprojection (MF-FBP) [28], [30], [33]. The MF-FBP approach introduced in [30], [34], and [33], however, requires knowledge of transmitter location, waveform and antenna beam pattern, and assumes that the received signal $d_{i}(t, s)$ in (4) can be decomposed into the components $d_{i, \mathbf{y}}(t, s)$ due to each transmitter. In this paper, we introduce a new image reconstruction method that uses (4) as the received signal model and does not necessarily require the knowledge of transmitter location, waveform or beam pattern. This method reconstructs the scene radiance, a quantity associated with the scene reflectivity, which we will introduce in the next section.

\section{Spatio-Temporal Correlation of Received Signals}

We address the image reconstruction problem for two different cases: reconstruction in the presence of i) cooperative sources of opportunity, where the information (transmitter location, waveform, antenna beam pattern, etc.,) about the sources are available, and ii) noncooperative sources of opportunity where such information is not available. We will first develop a model that connects the correlated measurements with the scene radiance, and then introduce the filtered-backprojection method.

We define the spatio-temporal correlation of $d_{i}$ and $d_{j}$ by

$$
d_{i j}\left(t, s, s^{\prime}\right)=\int d_{i}(s, \tau) d_{j}^{*}\left(\tau-t, s+s^{\prime}\right) d \tau
$$

where * denotes complex conjugation and $s^{\prime}$ is the slow-time delay. For the rest of the manuscript, we use $d_{i j}\left(t, s, s^{\prime}\right), s, s^{\prime} \in$ $\left[s_{a}, s_{b}\right], t \in\left[0, t_{0}\right], i, j=1, \ldots, N$, to reconstruct an image of the scene.

Note that for a single receiver $(N=1) d_{i j}=d_{11}$. We will reconstruct images for each $i, j$ pair for a range of $s, s^{\prime}$ and $t$ and sum all images to form the final image.

\section{A. Noncooperative Sources of Opportunity}

If the sources of opportunity are noncooperative, we use a stochastic model for the unknown terms, namely the transmitter antenna beam pattern $J_{T}$ and the scene reflectivity $G$.

Let $C_{G}$ and $C_{J_{T}}$ denote the correlation function of $G$ and $J_{T}$, respectively

$$
\begin{aligned}
C_{G}\left(\boldsymbol{x}, \boldsymbol{x}^{\prime}\right) & =E\left[G(\boldsymbol{x}) G^{*}\left(\boldsymbol{x}^{\prime}\right)\right] \\
C_{J_{T}}\left(\omega, \boldsymbol{x}, \boldsymbol{x}^{\prime}, \mathbf{y}, \mathbf{y}^{\prime}\right) & =E\left[J_{T}(\omega, \boldsymbol{x}, \mathbf{y}) J_{T}^{*}\left(\omega, \boldsymbol{x}^{\prime}, \mathbf{y}^{\prime}\right)\right]
\end{aligned}
$$

where $*$ denotes complex conjugate. We make the assumption that scene reflectivity $G$ and the transmit antenna beam pattern 
$J_{T}$ are statistically independent. Next, we make the incoherentfield approximation [35] by assuming that $G$ and $J_{T}$ satisfy the following equalities:

$$
\begin{aligned}
C_{G}\left(\boldsymbol{x}, \boldsymbol{x}^{\prime}\right) & =R_{G}(\boldsymbol{x}) \delta\left(\boldsymbol{x}-\boldsymbol{x}^{\prime}\right) \\
C_{\boldsymbol{J}_{T}}\left(\omega, \boldsymbol{x}, \boldsymbol{x}, \mathbf{y}, \mathbf{y}^{\prime}\right) & =R_{T}(\omega, \boldsymbol{x}, \mathbf{y}) \delta\left(\mathbf{y}-\mathbf{y}^{\prime}\right) .
\end{aligned}
$$

Here, $R_{G}$ is referred to as the scene radiance and $R_{T}$ as the transmitter irradiance (see page 525 of [35] for a definition of irradiance). Note that $R_{T}$ is the average power of electromagnetic radiation emitted by the transmitter at location $\mathbf{y}$ that is incident on the target surface at $\mathbf{x}$.

Plugging (1) and (4) into (5), performing the $\tau$ integration, assuming that $G$ and $J_{T}$ are statistically independent, and using (8) and (9), we find that the expected value of the correlated signal can be expressed as

$$
\begin{aligned}
E\left[d_{i j}\right. & \left.\left(t, s, s^{\prime}\right)\right] \\
= & \int \mathrm{e}^{-\mathrm{i} 2 \pi \omega\left(t-\left[\left|\mathbf{x}-\gamma_{R_{i}}(s)\right|-\left|\mathbf{x}^{\prime}-\boldsymbol{\gamma}_{R_{j}}\left(s+s^{\prime}\right)\right|\right] / c_{0}\right)} \\
& \times\left[\int \mathrm{e}^{\mathrm{i} 2 \pi \omega\left(\left[|\mathbf{y}-\mathbf{x}|-\left|\mathbf{y}^{\prime}-\mathbf{x}^{\prime}\right|\right] / c_{0}\right)}\right. \\
& \left.\times \frac{R_{T}(\omega, \boldsymbol{x}, \mathbf{y}) \delta\left(\mathbf{y}-\mathbf{y}^{\prime}\right)}{|\mathbf{x}-\mathbf{y}|\left|\mathbf{x}^{\prime}-\mathbf{y}^{\prime}\right|} d \mathbf{y} d \mathbf{y}^{\prime}\right] \\
& \times \frac{\omega^{2} \pi}{2} A_{R_{i j}}\left(\omega, s, s^{\prime}, \boldsymbol{x}, \boldsymbol{x}^{\prime}\right) \\
& \times R_{G}(\boldsymbol{x}) \delta\left(\boldsymbol{x}-\boldsymbol{x}^{\prime}\right) d \omega d \boldsymbol{x} d \boldsymbol{x}^{\prime} i, j=1, \ldots, N
\end{aligned}
$$

where

$$
\begin{aligned}
& A_{R_{i j}}\left(\omega, s, s^{\prime}, \boldsymbol{x}, \boldsymbol{x}^{\prime}\right) \\
& \quad=A_{R_{i}}(\omega, s, \boldsymbol{x}) A_{R_{j}}^{*}\left(\omega, s+s^{\prime}, \boldsymbol{x}^{\prime}\right) \\
& \quad i, j=1, \ldots, N .
\end{aligned}
$$

Carrying out the $\boldsymbol{x}^{\prime}$ and $\mathbf{y}^{\prime}$ integrations in (10), we note that the dependence of the phase on the transmitter locations $\mathbf{y}$ and $\mathbf{y}^{\prime}$ disappear, and we have simply

$$
\begin{aligned}
E\left[d_{i j}\left(t, s, s^{\prime}\right)\right] & \\
= & \mathcal{F}\left[R_{G}\right]\left(t, s, s^{\prime}\right) \\
:= & \int \mathrm{e}^{-\mathrm{i} 2 \pi \omega\left(t-r_{i j}\left(s, s^{\prime}, \mathbf{x}\right) / c_{0}\right)} A_{R_{i j}}\left(\omega, s, s^{\prime}, \boldsymbol{x}, \boldsymbol{x}\right) \\
& \times \tilde{R}_{T}(\omega, \boldsymbol{x}) R_{G}(\boldsymbol{x}) d \omega d \boldsymbol{x}, \quad i, j=1, \ldots, N
\end{aligned}
$$

where

$$
\begin{aligned}
\tilde{R}_{T}(\omega, \boldsymbol{x}) & =\frac{\omega^{2} \pi}{2} \int \frac{R_{T}(\omega, \boldsymbol{x}, \mathbf{y})}{|\mathbf{x}-\mathbf{y}|^{2}} d \mathbf{y} \\
& \text { and } \\
r_{i j}\left(s, s^{\prime}, \mathbf{x}\right) & =\left|\mathbf{x}-\gamma_{R_{i}}(s)\right|-\left|\mathbf{x}-\gamma_{R_{j}}\left(s+s^{\prime}\right)\right| .
\end{aligned}
$$

We will refer to $r_{i j}$ as the hitchhiker range. Note that $\tilde{R}_{T}(\omega, \boldsymbol{x})$ is the total average power incident upon the ground surface at $x$ due to all the transmitters. Therefore, we will refer to $\tilde{R}_{T}(\omega, \boldsymbol{x})$ as the total average transmitter irradiance.

\section{B. Cooperative Sources of Opportunity}

If the sources of opportunity are cooperative, we use a stochastic model for unknown scene reflectivity $G$; however, we assume that we have the full knowledge of transmitter locations and beam patterns. Thus, we treat $J_{T}$ deterministically.

The expected value of the correlated signal is then

$$
\begin{aligned}
& E\left[d_{i j}\left(t, s, s^{\prime}\right)\right] \\
& =\int \mathrm{e}^{-\mathrm{i} 2 \pi \omega\left(t-\left[\left|\mathbf{x}-\boldsymbol{\gamma}_{R_{i}}(s)\right|-\left|\mathbf{x}^{\prime}-\boldsymbol{\gamma}_{R_{j}}\left(s+s^{\prime}\right)\right|\right] / c_{0}\right)} \\
& \quad \times A_{R_{i j}}\left(\omega, s, s^{\prime}, \boldsymbol{x}, \boldsymbol{x}^{\prime}\right) B_{T}\left(\omega, \boldsymbol{x}, \boldsymbol{x}^{\prime}\right) \\
& \quad \times C_{G}\left(\boldsymbol{x}, \boldsymbol{x}^{\prime}\right) d \omega d \boldsymbol{x} d \boldsymbol{x}^{\prime}, i, j=1, \ldots, N
\end{aligned}
$$

where $C_{G}$ is the autocorrelation of $G$ given by (6), $A_{R_{i j}}$ is as in (11) and

$$
\begin{aligned}
B_{T}\left(\omega, \boldsymbol{x}, \boldsymbol{x}^{\prime}\right) & =\left[\int \mathrm{e}^{\mathrm{i} 2 \pi \omega|\mathbf{y}-\mathbf{x}| / c_{0}} A_{T}(\omega, \boldsymbol{x}, \mathbf{y}) d \mathbf{y}\right] \\
& \times\left[\int \mathrm{e}^{\mathrm{i} 2 \pi \omega\left|\mathbf{y}^{\prime}-\mathbf{x}^{\prime}\right| / c_{0}} A_{T}\left(\omega, \boldsymbol{x}^{\prime}, \mathbf{y}^{\prime}\right) d \mathbf{y}^{\prime}\right]^{*} .
\end{aligned}
$$

Next, we make the incoherent-field approximation (8) to (15), and simplify (15) to

$$
\begin{aligned}
E[ & \left.d_{i j}\left(t, s, s^{\prime}\right)\right] \\
= & \mathcal{F}\left[R_{G}\right]\left(t, s, s^{\prime}\right) \\
:= & \int \mathrm{e}^{-\mathrm{i} 2 \pi \omega\left(t-r_{i j}\left(s, s^{\prime}, \mathbf{x}\right) / c_{0}\right)} A_{R_{i j}}\left(\omega, s, s^{\prime}, \boldsymbol{x}, \boldsymbol{x}\right) \\
& \times \tilde{R}_{T}(\omega, \boldsymbol{x}) R_{G}(\boldsymbol{x}) d \omega d \boldsymbol{x}, \quad i, j=1, \ldots, N
\end{aligned}
$$

where $\tilde{R}_{T}(\omega, \boldsymbol{x})=B_{T}(\omega, \boldsymbol{x}, \boldsymbol{x}), r_{i j}$ is the hitchhiker range, and $\mathcal{F}$ is referred to as the forward modeling operator.

Note that (17) and (12) are exactly of the same with the exception that $\tilde{R}_{T}$ in (17) is given as a function of the deterministic transmit antenna beam pattern, whereas in (12) $\tilde{R}_{T}$ is given as a function of the autocorrelation of the transmit antenna beam pattern. Thus, the inversion formula for (17) and (12) will be the same.

\section{IMAGE Formation VIA C-FBP}

In the presence of sources of opportunity, our objective is to reconstruct $R_{G}$ given $E\left[d_{i j}\left(t, s, s^{\prime}\right)\right]$ for some range of $s, s^{\prime}$ and $t$ using the model (17) and (12).

We assume that for some $m_{A_{R_{i j}}}, A_{R_{i j}}$ and $\tilde{R}_{T}$ in (17) and (12) satisfy

$$
\begin{aligned}
& \sup _{\left(s, s^{\prime}, \boldsymbol{x}\right) \in K}\left|\partial_{\omega}^{\alpha} \partial_{s}^{\beta} \partial_{s^{\prime}}^{\beta^{\prime}} \partial_{x_{1}}^{\rho_{1}} \partial_{x_{2}}^{\rho_{2}} A_{R_{i j}}\left(\omega, s, s^{\prime}, \boldsymbol{x}, \boldsymbol{x}\right) \tilde{R}_{T}(\omega, \boldsymbol{x})\right| \\
& \leq C_{A}\left(1+\omega^{2}\right)^{\left(m_{A_{R_{i j}}}-|\alpha|\right) / 2}
\end{aligned}
$$

where $K$ is any compact subset of $\mathbb{R} \times \mathbb{R} \times \mathbb{R}^{2}$, and the constant $C_{A}$ depends on $K, \alpha, \beta, \beta^{\prime}, \rho_{1}$, and $\rho_{2}$. This assumption is needed in order to make various stationary phase calculations hold. In practice, (18) is satisfied for transmitters and receivers sufficiently away from the illuminated region. This is the case especially for air-/space-borne transmitters and receivers, and broadcasting stations located on high grounds. 


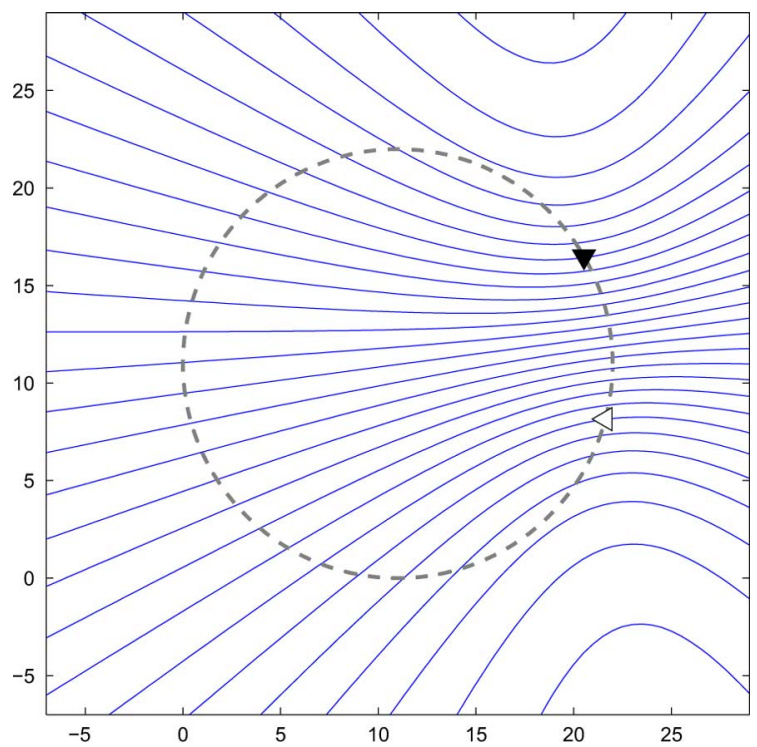

Fig. 2. Iso-range contours $\left(H_{i j}\left(t, s, s^{\prime}\right)\right)$ for the hitchhiker range $r_{11}(-\pi / 8, \pi / 4, \boldsymbol{x})$ of a circular flight trajectory (dashed line) $\gamma_{R_{1}}(s)=$ $\gamma_{C}(s)$, over a flat topography where white and black triangles are $\gamma_{C}(-\pi / 8)$ and $\gamma_{C}(\pi / 8)$, respectively. (see (14) and Section VI, Fig. 5, for explicit formulae of $r_{i j}\left(s, s^{\prime}, \boldsymbol{x}\right)$ and $\boldsymbol{\gamma}_{C}(s)$, respectively).

Under the assumption (12), (17), and (18) define $\mathcal{F}$ as a Fourier integral operator [36] whose leading-order contribution comes from those points lying in the intersection of the illuminated surface and the hyperboloid $\left\{\mathbf{x} \in \mathbb{R}^{3}: r_{i j}\left(s, s^{\prime}, \mathbf{x}\right)=c_{0} t\right\}$. We denote the curves formed by this intersection by $H_{i j}\left(t, s, s^{\prime}\right)=\left\{\boldsymbol{x}: r_{i j}\left(s, s^{\prime}, \mathbf{x}\right)=c_{0} t\right\}$ and refer to $H_{i j}\left(t, s, s^{\prime}\right)$ as an iso-range contour. For flat topography, $\psi(\boldsymbol{x})=0$, the iso-range contours are given by hyperbolas on the plane $x_{3}=0$. For circular receiver flight trajectories over flat topography we present the iso-range contours in Fig. 2. Thus, our inverse problem is to reconstruct the target scene radiance $R_{G}$ from the averaged measurements $E\left[d_{i j}\right]$ by inverting the Fourier integral operator $\mathcal{F}$ given in (17) or (12).

Note that many sources of opportunity, such as satellite communications and cell-phone towers, transmit multiple pulses to combat noise. Therefore, one method of obtaining an estimate of $E\left[d_{i j}\right]$ is to average $d_{i j}$ 's over multiple pulses received at every point on the flight trajectory. Alternatively, $d_{i j}$ itself can serve as an estimate of $E\left[d_{i j}\right]$.

\section{A. Filtered-Backprojection Operator}

Since $\mathcal{F}$ is a Fourier integral operator, an approximate inversion of $\mathcal{F}$ can be computed by a suitable filtered-backprojection operation [36]

$$
\begin{aligned}
& \mathcal{K}\left[\mathcal{F}\left[R_{G}\right]\right](\boldsymbol{z}) \\
& =\sum_{i j} \int \mathrm{e}^{\mathrm{i} 2 \pi \omega\left(t-r_{i j}\left(s, s^{\prime}, \mathbf{z}\right) / c_{0}\right)} Q_{i j}\left(z, \omega, s, s^{\prime}\right) \\
& \quad \times \mathcal{F}\left[R_{G}\right]\left(t, s, s^{\prime}\right) d t d \omega d s d s^{\prime}
\end{aligned}
$$

where $\mathcal{K}$ will be referred to as the filtered-backprojection operator with $Q_{i j}$ being the filter to be determined. We will refer to the special case of $Q_{i j}=1$ (19) as correlation-backprojection (C-BP) reconstruction.
We should note that for some $m_{Q_{i j}}, Q_{i j}$ must satisfy

$$
\begin{aligned}
\sup _{\left(s, s^{\prime}, z\right) \in K} \mid \partial_{\omega}^{\alpha} \partial_{s}^{\beta} \partial_{s^{\prime}}^{\beta^{\prime}} \partial_{z_{1}}^{\rho_{1}} \partial_{z_{2}}^{\rho_{2}} & Q_{i j}\left(\boldsymbol{z}, \omega, s, s^{\prime}\right) \mid \\
& \leq C_{Q}\left(1+\omega^{2}\right)^{\left(m_{Q_{i j}}-|\alpha|\right) / 2}
\end{aligned}
$$

where $K$ is any compact subset of $\mathbb{R} \times \mathbb{R} \times \mathbb{R}^{2}$, and the constant $C_{Q}$ depends on $K, \alpha, \beta, \beta^{\prime}, \rho_{1}$, and $\rho_{2}$.

Substituting (17) into (19), we approximate $R_{G}$ by

$$
\hat{R}_{G}(z)=\mathcal{K} \mathcal{F}\left[R_{G}\right](z)=\int L(z, x) R_{G}(\boldsymbol{x}) d \boldsymbol{x}
$$

where

$$
\begin{aligned}
L(\boldsymbol{z}, \boldsymbol{x})= & \sum_{i j} \int \mathrm{e}^{\mathrm{i} 2 \pi \omega\left[r_{i j}\left(s, s^{\prime}, \mathbf{x}\right)-r_{i j}\left(s, s^{\prime}, \mathbf{z}\right)\right] / c_{0}} \\
& \times Q_{i j}\left(z, \omega, s, s^{\prime}\right) A_{i j}\left(\omega, s, s^{\prime}, \boldsymbol{x}, \boldsymbol{x}\right) d \omega d s d s^{\prime}
\end{aligned}
$$

is the point spread function, and $A_{i j}=A_{R_{i j}} \tilde{R}_{T}$.

Under the assumptions (18) and (20), $\mathcal{K}$ produces an approximation $\hat{R}_{G}$ to $R_{G}$. As we see in the next section, any nonzero choice of filter leads to an $\hat{R}_{G}$ whose visible edges of $R_{G}$ are at the correct location and orientation. However, not all filters reconstruct the visible edges with correct amplitude [28], [29], [37], [38].

The filter $Q_{i j}$ can be determined with respect to various criteria [38]. Here, we will determine $Q_{i j}$ so that the point spread function of the resulting imaging operator is approximately a Dirac delta function, $\delta(\boldsymbol{x}-\boldsymbol{z})=\int \exp (\mathrm{i} 2 \pi(\boldsymbol{x}-\boldsymbol{z}) \cdot \boldsymbol{\xi}) d \boldsymbol{\xi}$. This choice of $Q_{i j}$ reconstructs visible edges with correct amplitude [28], [29], [37], [38].

\section{B. Determination of the FBP Filter}

Let us write

$$
L(z, \boldsymbol{x})=\sum_{i j} \int L_{i j}\left(z, x, s^{\prime}\right) d s^{\prime}
$$

where

$$
\begin{aligned}
L_{i j}\left(\boldsymbol{z}, \boldsymbol{x}, s^{\prime}\right)= & \left.\int_{Q_{i j}} \mathrm{e}^{\mathrm{i} 2 \pi \omega\left[r_{i j}\left(s, s^{\prime}, \mathbf{x}\right)-r_{i j}\left(s, s^{\prime}, \mathbf{z}\right)\right] / c_{0}}, s^{\prime}\right) \times A_{i j}\left(\omega, s, s^{\prime}, \boldsymbol{x}, \boldsymbol{x}\right) d \omega d s .
\end{aligned}
$$

We will determine the filter $Q_{i j}$ such that $L_{i j}\left(\boldsymbol{z}, \boldsymbol{x}, s^{\prime}\right)$ is as close as possible to the Dirac delta function $(\delta(\boldsymbol{z}-\boldsymbol{x}))$, so that the leading order contribution to the point spread function is the sum of Dirac delta functions.

Applying the method of stationary phase (see Appendix A) to the $\omega$ and $s$ integrals, we find that the main contribution to $L_{i j}\left(\boldsymbol{z}, \boldsymbol{x}, s^{\prime}\right)$ come from those critical points of its phase. The critical points satisfy the conditions

$$
\begin{aligned}
& \partial_{\omega}\left(\omega\left[r_{i j}\left(s, s^{\prime}, \mathbf{x}\right)-r_{i j}\left(s, s^{\prime}, \mathbf{z}\right)\right] / c_{0}\right)=0 \\
& \Longrightarrow r_{i j}\left(s, s^{\prime}, \mathbf{x}\right)=r_{i j}\left(s, s^{\prime}, \mathbf{z}\right) \\
& \partial_{s}\left(\omega\left[r_{i j}\left(s, s^{\prime}, \mathbf{x}\right)-r_{i j}\left(s, s^{\prime}, \mathbf{z}\right)\right] / c_{0}\right)=0 \\
& \Longrightarrow f_{i j}\left(s, s^{\prime}, \mathbf{x}\right)=f_{i j}\left(s, s^{\prime}, \mathbf{z}\right),
\end{aligned}
$$




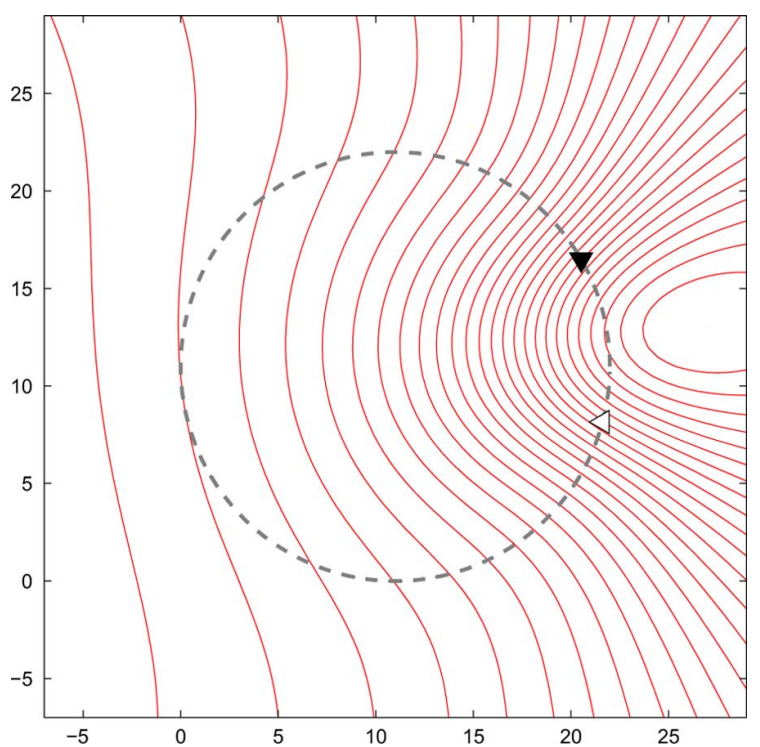

Fig. 3. Iso-Doppler contours $F_{i j}\left(s, s^{\prime}, C\right)$ for the hitchhiker Doppler $f_{11}(-\pi / 8, \pi / 4, \mathbf{x})$ of a circular flight trajectory (dashed line) $\boldsymbol{\gamma}_{R_{1}}(s)=$ $\gamma_{C}(s)$, over a flat topography where white and black triangles are $\gamma_{C}(-\pi / 8)$ and $\gamma_{C}(\pi / 8)$, respectively [see (27) and Section VI, Fig. 5, for explicit formulae of the hitchhiker Doppler $f_{i j}\left(s, s^{\prime}, \mathbf{x}\right)$ and $\gamma_{C}(s)$, respectively].

where

$$
\begin{aligned}
f_{i j}\left(s, s^{\prime}, \mathbf{x}\right)=\frac{\omega}{c_{0}} & {\left[\dot{\gamma}_{R_{i}}(s) \cdot \frac{\gamma_{R_{i}}(s)-\mathbf{x}}{\left|\gamma_{R_{i}}(s)-\mathbf{x}\right|}\right.} \\
& \left.\quad \dot{\gamma}_{R_{j}}\left(s+s^{\prime}\right) \cdot \frac{\gamma_{R_{j}}\left(s+s^{\prime}\right)-\mathbf{x}}{\left|\gamma_{R_{j}}\left(s+s^{\prime}\right)-\mathbf{x}\right|}\right] .
\end{aligned}
$$

We will refer to $f_{i j}$ as the hitchhiker Doppler. Here $\dot{\gamma}_{R_{i}, R_{j}}(s)=$ $\partial_{s} \gamma_{R_{i}, R_{j}}(s)$ is the partial derivative of the trajectories with respect to $s$. Thus, the hitchhiker Doppler is defined by the difference between the radial velocities of the receivers $R_{i}$ and $R_{j}$ with respect to the point $\mathbf{x}$ divided by the wavelength $c_{0} / \omega$.

For each $\left(s, s^{\prime}\right)$, we refer to the locus of points that is formed by the intersection of the surface topography and $\left\{\mathbf{x} \in \mathbb{R}^{3}\right.$ : $\left.f_{i j}\left(s, s^{\prime}, \mathbf{x}\right)=C\right\}$, for some constant $C$, as an iso-Doppler contour. We denote an iso-Doppler contour by $F_{i j}\left(s, s^{\prime}, C\right)=\{\boldsymbol{x}$ : $\left.f_{i j}\left(s, s^{\prime}, \mathbf{x}\right)=C\right\}$. Fig. 3 shows the iso-Doppler contours for circular flight trajectory and flat topography.

The critical points $\mathbf{z}$ of the phase of $L_{s^{\prime}}$ are those points that have the same hitchhiker range and hitchhiker Doppler with $\mathbf{x}$. We assume that the flight trajectories of the receivers are smooth and that the receiver antenna beam patterns are focused on a region of interest where every pair of iso-range $\left(H_{i j}\left(t, s, s^{\prime}\right)\right)$ and iso-Doppler $\left(F_{i j}\left(s, s^{\prime}, C\right)\right)$ contours intersect at a single point within the region of interest. In other words, we assume that the only critical point within the region of interest is $\boldsymbol{z}=\boldsymbol{x}$.

Using the fundamental theorem of Calculus [39]

$$
\begin{aligned}
h(\boldsymbol{x}) & -h(\boldsymbol{z}) \\
= & \int_{0}^{1} \frac{\partial}{\partial \lambda} h(\boldsymbol{z}+\lambda(\boldsymbol{x}-\boldsymbol{z})) d \lambda
\end{aligned}
$$

$$
=(\boldsymbol{x}-\boldsymbol{z}) \cdot \int_{0}^{1} \nabla h(z+\lambda(\boldsymbol{x}-\boldsymbol{z})) d \lambda
$$

we write

$$
\left[r_{i j}\left(s, s^{\prime}, \mathbf{x}\right)-r_{i j}\left(s, s^{\prime}, \mathbf{z}\right)\right]=(\boldsymbol{x}-\boldsymbol{z}) \cdot \boldsymbol{\Xi}_{i j}\left(s, s^{\prime}, \boldsymbol{x}, \boldsymbol{z}\right)
$$

where

$$
\boldsymbol{\Xi}_{i j}\left(s, s^{\prime}, \boldsymbol{x}, \boldsymbol{z}\right)=\int_{0}^{1} \nabla r_{i j}\left(s, s^{\prime}, \boldsymbol{z}+\lambda(\boldsymbol{x}-\boldsymbol{z})\right) d \lambda .
$$

For fixed $s^{\prime}, \boldsymbol{x}$ and $\boldsymbol{z}$, we make the following change of variables:

$$
(\omega, s) \rightarrow \boldsymbol{\xi}_{i j}=\frac{\omega}{c_{0}} \boldsymbol{\Xi}_{i j}\left(s, s^{\prime}, \boldsymbol{x}, \boldsymbol{z}\right)
$$

in the integral of (22) to obtain the point spread function as

$$
\begin{aligned}
L(\boldsymbol{z}, \boldsymbol{x})=\sum_{i j} & \int \mathrm{e}^{\mathrm{i} 2 \pi(\boldsymbol{x}-\boldsymbol{z}) \cdot \boldsymbol{\xi}_{i j}} Q_{i j}\left(\boldsymbol{z}, \boldsymbol{\xi}_{i j}, s^{\prime}\right) \\
& \times A_{i j}\left(\boldsymbol{\xi}_{i j}, s^{\prime}, \boldsymbol{x}, \boldsymbol{x}\right) \eta\left(\boldsymbol{x}, z, \boldsymbol{\xi}_{i j}, s^{\prime}\right) d \boldsymbol{\xi}_{i j} d s^{\prime}
\end{aligned}
$$

where $Q_{i j}\left(z, \boldsymbol{\xi}_{i j}, s^{\prime}\right)=Q_{i j}\left(\boldsymbol{z}, \omega\left(\boldsymbol{\xi}_{i j}, s\left(\boldsymbol{\xi}_{i j}\right)\right), s^{\prime}\right)$, and $\eta\left(\boldsymbol{x}, z, \boldsymbol{\xi}_{i j}, s^{\prime}\right)=\left|\partial(\omega, s) / \partial \boldsymbol{\xi}_{i j}\right|$, is the determinant of the Jacobian that comes from the change of variables (32).

It can be shown, by treating (33) as a distribution and using the method of stationary phase, that under the assumption (18), the leading-order contribution to $\hat{R}_{G}$ is at $z=x$. Consequently, we approximate (33) as

$$
\begin{aligned}
\hat{R}_{G}(\boldsymbol{z}) \approx & \sum_{i j} \int_{\Omega_{i j}, \boldsymbol{z}, s^{\prime}} \mathrm{e}^{\mathrm{i} 2 \pi(\boldsymbol{x}-\boldsymbol{z}) \cdot \boldsymbol{\xi}_{i j}} \\
& \times Q_{i j}\left(\boldsymbol{z}, \boldsymbol{\xi}_{i j}, s^{\prime}\right) \times A_{i j}\left(\boldsymbol{\xi}_{i j}, s^{\prime}, \boldsymbol{z}, \boldsymbol{z}\right) \\
& \times \eta\left(\boldsymbol{z}, \boldsymbol{z}, \boldsymbol{\xi}_{i j}, s^{\prime}\right) R_{G}(\boldsymbol{x}) d \boldsymbol{x} d \boldsymbol{\xi}_{i j} d s^{\prime}
\end{aligned}
$$

where

$$
\begin{aligned}
\Omega_{i j, \boldsymbol{z}, s^{\prime}}=\left\{\boldsymbol{\xi}_{i j}=\right. & \omega / c_{0} \boldsymbol{\Xi}_{i j}\left(s, s^{\prime}, \boldsymbol{z}, \boldsymbol{z}\right) \mid \\
& \left.A_{i j}\left(\omega, s, s^{\prime}, \boldsymbol{z}, \boldsymbol{z}\right) \neq 0, \quad s \in\left[s_{a}, s_{b}\right]\right\}
\end{aligned}
$$

with

$$
\begin{aligned}
& \boldsymbol{\Xi}_{i j}\left(s, s^{\prime}, \boldsymbol{z}, \boldsymbol{z}\right) \\
& \quad=D \psi(\boldsymbol{z}) \cdot\left[\frac{\boldsymbol{\gamma}_{R_{j}}\left(s+s^{\prime}\right)-\mathbf{z}}{\left|\boldsymbol{\gamma}_{R_{j}}\left(s+s^{\prime}\right)-\mathbf{z}\right|}-\frac{\gamma_{R_{i}}(s)-\mathbf{z}}{\left|\gamma_{R_{i}}(s)-\mathbf{z}\right|}\right] .
\end{aligned}
$$

Here

$$
D \psi(z)=\left[\begin{array}{lll}
1 & 0 & \partial \psi(\boldsymbol{z}) / \partial z_{1} \\
0 & 1 & \partial \psi(\boldsymbol{z}) / \partial z_{2}
\end{array}\right]
$$

For flat topography, we present an illustration of $\boldsymbol{\Xi}_{i j}\left(s, s^{\prime}, \mathbf{z}, \mathbf{z}\right)$ in Fig. 4.

Thus, with the choice of

$$
Q_{i j}\left(\boldsymbol{z}, \omega, s, s^{\prime}\right)=\frac{\chi_{\Omega_{i j, \boldsymbol{z}, s^{\prime}}}\left(\boldsymbol{\xi}_{i j}(\omega, s)\right)}{\eta\left(\boldsymbol{z}, \boldsymbol{z}, \boldsymbol{\xi}_{i j}, s^{\prime}\right)} \frac{A_{i j}^{*}\left(\omega, s, s^{\prime}, \boldsymbol{z}, \boldsymbol{z}\right)}{\left|A_{i j}\left(\omega, s, s^{\prime}, \boldsymbol{z}, \boldsymbol{z}\right)\right|^{2}}
$$




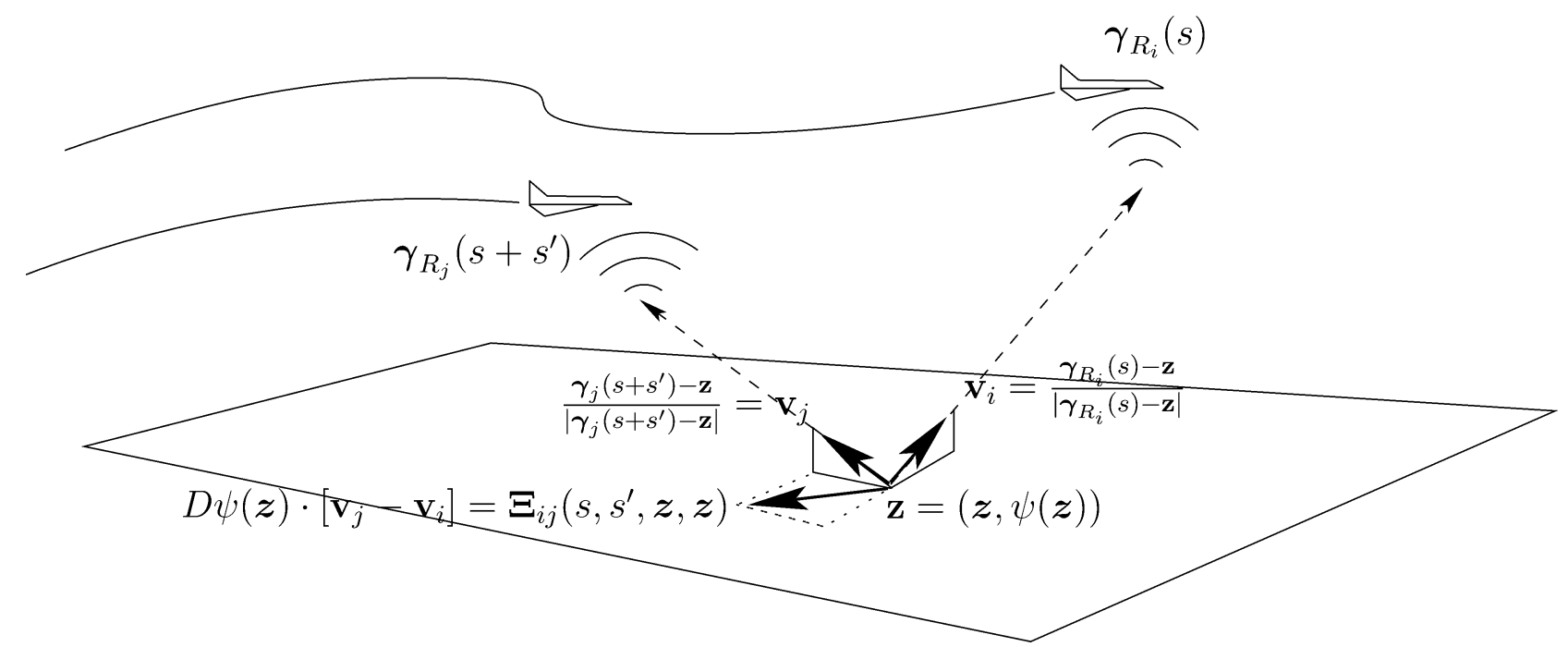

Fig. 4. Illustration of the vector $\boldsymbol{\Xi}_{i j}\left(s, s^{\prime}, \boldsymbol{z}, \boldsymbol{z}\right)$ in the data collection manifold $\Omega_{i j, \boldsymbol{z}, s^{\prime}}$ for flat topography, $\psi(\boldsymbol{z})=0$. $\boldsymbol{\Xi}_{i j}\left(s, s^{\prime}, \boldsymbol{z}, \boldsymbol{z}\right)$ is the projection of the difference of the vectors $\mathbf{v}_{i}$ and $\mathbf{v}_{j}$ onto the tangent plane at location $\mathbf{z}$ (see (36) for explicit formula of $\mathbf{\Xi}\left(s, s^{\prime}, \boldsymbol{z}, \boldsymbol{z}\right)$ ).

where $\chi_{\Omega_{i j}, \boldsymbol{Z}, s^{\prime}}$ is a smooth cut-off function equal to one in most of the interior of $\Omega_{i j, z, s^{\prime}}$ and zero in the exterior of $\Omega_{i j, \boldsymbol{z}, s^{\prime}},(34)$ becomes

$$
\begin{aligned}
\hat{R}_{G}(\boldsymbol{z}) \approx \sum_{i j} \int \mathrm{e}^{\mathrm{i}(\boldsymbol{x}-\boldsymbol{z}) \cdot \boldsymbol{\xi}_{i j}} \chi_{\Omega_{i j}, \boldsymbol{z}, s^{\prime}}\left(\boldsymbol{\xi}_{i j}\right) & \\
\times & R_{G}(\boldsymbol{x}) d \boldsymbol{x} d \boldsymbol{\xi}_{i j} d s^{\prime} .
\end{aligned}
$$

Equation (39) shows that the image $\hat{R}_{G}$ is a band-limited version of $R_{G}$ whose frequency content, by (32), is determined by $\Omega_{z}=\bigcup_{i j, s^{\prime}} \Omega_{i j, z, s^{\prime}}$. We will refer to $\Omega_{z}$ as the data collection manifold at $z$. In this regard, we will refer to $\Omega_{i j, z, s^{\prime}}$ as the partial data collection manifold at $z$ obtained by $i$ th and $j$ th receiver pair at slow-time delay $s^{\prime}$.

Mircolocal analysis of (39) tells us that an edge at point $z$ is visible if the direction $\boldsymbol{n}_{\boldsymbol{z}}$ normal to the edge is contained in the data collection manifold $\Omega_{z}$ [28], [29], [37], [38]. Consequently, we say an edge at point $\boldsymbol{z}$ with $\boldsymbol{n}_{\boldsymbol{z}}$ normal to the edge is visible, if there exists $i, j, s$ and $s^{\prime}$, such that $\boldsymbol{\Xi}_{i j}\left(s, s^{\prime}, \boldsymbol{z}, \boldsymbol{z}\right)$ is parallel to $\boldsymbol{n}_{\boldsymbol{z}}$. We see from (39) that we can only reconstruct the edges of $R_{G}$ that are visible. Furthermore, with the choice of (38), we can reconstruct not only the correct location and orientation but also the correct amplitude of the visible edges [28], [29], [38].

In the case of noncooperative sources of opportunity, if $\tilde{R}_{T}$ is not available, we may assume that the scene is homogeneously illuminated by isotropic transmitters and set $\tilde{R}_{T}=1$. Note that this assumption can be interpreted as an uninformative prior on the transmit antenna beam patterns. As a consequence $A_{i j}$ will be equal to $A_{R_{i j}}$. With this choice of filter we are still able reconstruct the visible edges at the correct location and orientation but not with correct amplitude [28], [29], [37], [38]. Thus, the reconstruction formula becomes

$$
\begin{aligned}
\mathcal{K}\left[d_{i j}\right](\mathbf{z})= & \sum_{i j} \int \mathrm{e}^{\mathrm{i} 2 \pi \omega-r_{i j}\left(s, s^{\prime}, \mathbf{z}\right) / c_{0}} \\
& \times \frac{\chi_{\Omega_{i j}, \boldsymbol{z}, s^{\prime}}\left(\boldsymbol{\xi}_{i j}(\omega, s)\right)}{\eta\left(\boldsymbol{z}, \boldsymbol{z}, \boldsymbol{\xi}_{i j}, s^{\prime}\right)} \times \frac{A_{R_{i j}}^{*}\left(\omega, s, s^{\prime}, \boldsymbol{z}, \boldsymbol{z}\right)}{\left|A_{R_{i j}}\left(\omega, s, s^{\prime}, \boldsymbol{z}, \boldsymbol{z}\right)\right|^{2}} \\
& \times d_{i j}\left(t, s, s^{\prime}\right) d t d \omega d s d s^{\prime} .
\end{aligned}
$$

\section{Reconstruction Algorithm AND the Analysis of COMPUTATIONAL COMPLEXITY}

In this section, we present our numerical implementation of the C-FBP method, analyze its computational complexity.

We implemented the inversion formula (19) and (38).

Let $\boldsymbol{\Xi}_{i j}\left(s, s^{\prime}, \boldsymbol{z}, \boldsymbol{z}\right)=\left(\Xi_{i j, 1}, \Xi_{i j, 2}\right)$ and $\partial_{s} \boldsymbol{\Xi}_{i j}\left(s, s^{\prime}, \boldsymbol{z}, \boldsymbol{z}\right)=$ $\left(\dot{\Xi}_{i j, 1}, \dot{\Xi}_{i j, 2}\right)$. Then, by (32) and (36)

$$
\begin{aligned}
\frac{1}{\eta\left(z, z, \xi_{i j}, s^{\prime}\right)} & =\left|\frac{\partial \boldsymbol{\xi}_{i j}}{\partial(\omega, s)}\right| \\
& =\frac{|\omega|}{c_{0}^{2}}\left|\Xi_{i j, 1} \dot{\Xi}_{i j, 2}-\Xi_{i j, 2} \dot{\Xi}_{i j, 1}\right| .
\end{aligned}
$$

Then, we can rewrite the filter (38) as

$$
Q_{i j}\left(z, \omega, s, s^{\prime}\right)=Q_{i j, 1}\left(s, s^{\prime}, z\right) Q_{i j, 2}\left(\omega, s, s^{\prime}, z\right)|\omega|
$$

where

$$
\begin{aligned}
& Q_{i j, 1}\left(s, s^{\prime}, \boldsymbol{z}\right) \\
& \quad=\left|\Xi_{i j, 1} \dot{\Xi}_{i j, 2}-\Xi_{i j, 2} \dot{\Xi}_{i j, 1}\right| \\
& Q_{i j, 2}\left(\omega, s, s^{\prime}, \boldsymbol{z}\right) \\
& \quad=\frac{\chi_{\Omega_{i j}, \boldsymbol{z}, s^{\prime}}\left(\boldsymbol{\xi}_{i j}(\omega, s)\right)}{c_{0}^{2}} \frac{A_{i j}^{*}\left(\omega, s, s^{\prime}, z, z\right)}{\left|A_{i j}\left(\omega, s, s^{\prime}, \boldsymbol{z}, \boldsymbol{z}\right)\right|^{2}} .
\end{aligned}
$$

Additionally, we assume that a single realization of $d_{i}(t, s)$ [see (4)] is available and replace all $E\left[d_{i j}\right]$ 's in our reconstruction formulae with $d_{i j}$ 's.

Thus, using (19) and (38), our reconstruction formula becomes

$$
\begin{aligned}
\hat{R}_{G}(z)=\sum_{i j} & \int \mathrm{e}^{-\mathrm{i} 2 \pi \omega r_{i j}\left(s, s^{\prime}, \mathbf{z}\right) / c_{0}}|\omega| D_{i j}\left(\omega, s, s^{\prime}\right) \\
& \times Q_{i j, 1}\left(s, s^{\prime}, \boldsymbol{z}\right) Q_{i j, 2}\left(\omega, s, s^{\prime}, \boldsymbol{z}\right) d \omega d s d s^{\prime}
\end{aligned}
$$

where

$$
D_{i j}\left(\omega, s, s^{\prime}\right)=\int \mathrm{e}^{\mathrm{i} 2 \pi \omega t} d_{i j}\left(t, s, s^{\prime}\right) d t
$$


Assuming that there are $\mathcal{O}(N)$ samples in both fast-time and slow-time variables, and the scene is sampled at $\mathcal{O}(N \times N)$ points, for each $i$ and $j$, the steps of the reconstruction and their corresponding computational complexity are as follows.

1) Computing the Fourier Transform in Fast-time: For each $s$ and $s^{\prime}$, (46) can be computed using the fast Fourier transform (FFT) in $\mathcal{O}(N \log N)$ computations. Thus, for all $s$ and $s^{\prime}$, the computational complexity of this step is $\mathcal{O}\left(N^{3} \log N\right)$.

2) Ramp Filtering: In tomography literature, multiplication with $|\omega|$ in the Fourier domain is referred to as the Ram-Lak or the ramp filter [40]. Let

$$
\tilde{D}_{i j}\left(\omega, s, s^{\prime}\right)=|\omega| D_{i j}\left(\omega, s, s^{\prime}\right),
$$

be the Fourier transform of the ramp filtered measurements. For each $s$ and $s^{\prime}$, (48) can be computed in $\mathcal{O}(N)$ number of computations. Thus, for all $s$ and $s^{\prime}$, the computational complexity of the ramp filtering step is $\mathcal{O}\left(N^{3}\right)$.

3) Filtering with $Q_{i j, 2}$ : Let

$$
\tilde{D}_{i j, Q_{i j, 2}}\left(\omega, s, s^{\prime}, z\right)=\tilde{D}_{i j}\left(\omega, s, s^{\prime}\right) Q_{i j, 2}\left(\omega, s, s^{\prime}, z\right) .
$$

For each $s, s^{\prime}$ and $z$, (49) can be computed in $\mathcal{O}(N)$ number of computations. Thus, for all $s, s^{\prime}$ and $z$ the computational complexity of this step is $O\left(N^{5}\right)$. If $Q_{i j, 2}$ is independent of $\boldsymbol{z}$, then the computational complexity of this step reduces to $O\left(N^{3}\right)$.

4) Backprojection Step: Let

$$
\begin{aligned}
\hat{R}_{G_{i j, 2}}\left(s, s^{\prime}, z\right)=\int \mathrm{e}^{-\mathrm{i} 2 \pi \omega r_{i j}\left(s, s^{\prime}, \mathbf{z}\right) / c_{0}} & \\
& \times \tilde{D}_{i j, Q_{i j, 2}}\left(\omega, s, s^{\prime}, \boldsymbol{z}\right) d \omega .
\end{aligned}
$$

For each $s, s^{\prime}$ and $z$, (3) can be computed using FFT or direct computation in $\mathcal{O}(N \log N)$ and $\mathcal{O}(N)$ computations, respectively. Thus, for all $s, s^{\prime}$ and $z$, the computational complexity of this step is $\mathcal{O}\left(N^{5} \log N\right)$ using FFT and $\mathcal{O}\left(N^{5}\right)$ by direct computation. If $Q_{i j, 2}$ is independent of $z$ then the computational complexity of this step reduces to $\mathcal{O}\left(N^{3} \log N\right)$ using FFT and $\mathcal{O}\left(N^{5}\right)$ by direct computation.

5) Partial Image Formation: We form the partial image $\hat{R}_{G_{i j}}$ by

$$
\hat{R}_{G_{i j}}(\boldsymbol{z})=\int Q_{i j, 1}\left(s, s^{\prime}, z\right) \hat{R}_{G_{i j, 2}}\left(s, s^{\prime}, z\right) d s d s^{\prime} .
$$

The computational complexity of this step is $\mathcal{O}\left(N^{4}\right)$.

6) Complete Image Formation: Finally, we form our image $\hat{R}_{G}$ by summing over partial images

$$
\hat{R}_{G}(z)=\sum_{i j} \hat{R}_{G_{i j}}(z) .
$$

For each pair of $i$ and $j$, the computational complexity of our algorithm up to the complete image formation step is determined by the backprojection step when $Q_{i j, 2}$ is $z$ dependent $\left(\mathcal{O}\left(N^{5}\right)\right)$ or the partial image formation step when $Q_{i j, 2}$ is $z$ independent $\left(\mathcal{O}\left(N^{4}\right)\right)$. For flat topography and isotropic receiver antennas, $Q_{i j, 2}$ is independent of $z$. If both $Q_{i j, 1}$ and

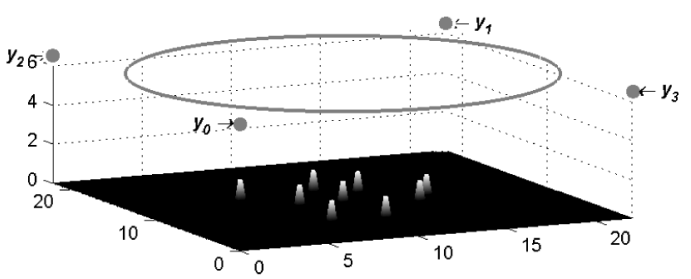

(a)

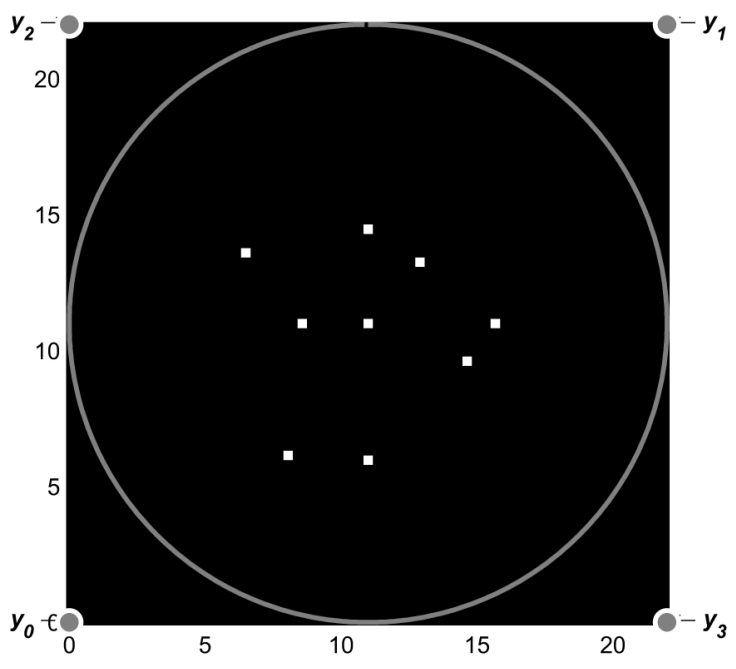

(b)

Fig. 5. (a) 3-D and (b) 2-D views of the scene with transmitters located at $\mathbf{y}_{0}=$ $(0,0,6.5) \mathrm{km}, \mathbf{y}_{1}=(22,22,6.5) \mathrm{km}, \mathbf{y}_{2}=(0,22,6.5) \mathrm{km}$, and $\mathbf{y}_{3}=$ $(22,0,6.5) \mathrm{km}$, and circular receiver trajectory (solid line) $\gamma_{C}(s)=(11+$ $11 \cos (s), 11+11 \sin (s), 6.5) \mathrm{km}$.

$Q_{i j, 2}$ are independent of $z$ then the computational complexity of $\mathrm{C}$-FBP up to the complete image formation step reduces down to $\mathcal{O}\left(N^{3} \log N\right)$, which is the case for C-BP reconstruction. The total computational complexity, including the complete image formation step, is $N^{2}$ times the computational complexity of the partial image-formation algorithm.

For the case of a single receiver antenna $(i=j=$ 1 ), although $\mathrm{C}-\mathrm{FBP}$ has a greater computational complexity $\left(\mathcal{O}\left(N^{3} \log N\right)-\mathcal{O}\left(N^{5}\right)\right)$ than that of MF-FBP $\left(\mathcal{O}\left(N^{2} \log N\right)-\mathcal{O}\left(N^{3}\right)\right)$ [34], C-FBP does not require the knowledge of the transmitter locations or other transmitter related information, and can, therefore, be used for multiple, noncooperative sources of opportunity.

\section{NUMERICAL SimULATIONS}

In this section, we demonstrate the performance of our method in numerical simulations under variety of operating conditions and assumptions. These include the following cases: a) single transmitter and single receiver, b) multiple transmitters and single receiver, c) single transmitter and multiple receivers, and d) multiple transmitters and multiple receivers. For the single-receiver case, we consider both cooperative and noncooperative sources of opportunity. For the multiple-receiver case, to keep our discussion brief, we consider only noncooperative sources of opportunity. The C-FBP reconstructions are compared with the $\mathrm{C}-\mathrm{BP}$ reconstructions for all cases. 


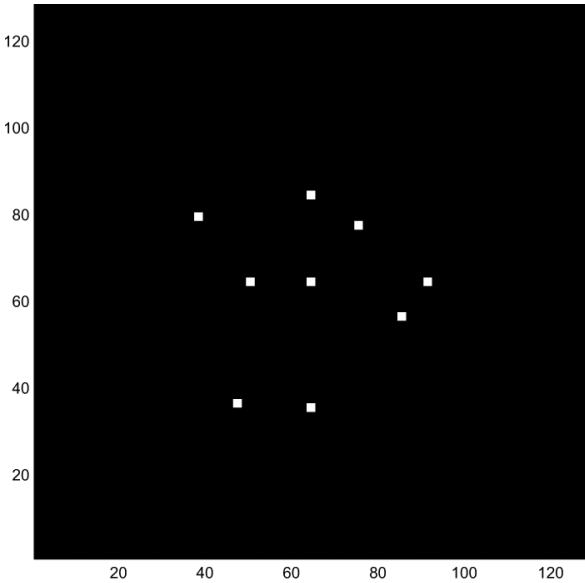

(a)
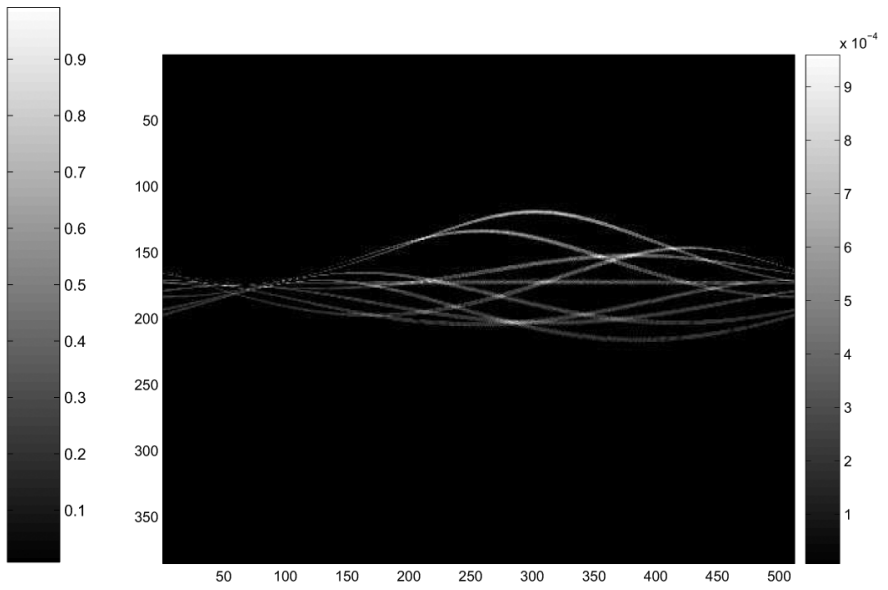

(b)

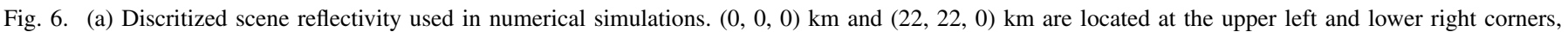

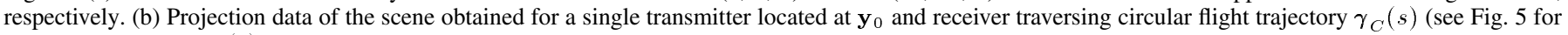
explicit formula of $\gamma_{C}(s)$ ).

In accordance with the incoherent field approximation, we consider the following idealized target model for the scene reflectivity

$$
G(\boldsymbol{x})=\sum_{n=1}^{N} g_{n} \delta\left(\boldsymbol{x}-\boldsymbol{x}_{n}\right)
$$

where $\left\{g_{n}\right\}$ are independent normal random variables with mean $\mu_{n}$ and variance $\sigma_{n}^{2}$. The corresponding scene radiance is given by

$$
R_{G}(x)=E\left[G(x) G^{*}(x)\right]=\sum_{n}\left(\mu_{n}^{2}+\sigma_{n}^{2}\right) \delta\left(x-x_{n}\right)
$$

For most of our simulations, we consider a deterministic reflectivity and set $\sigma_{n}^{2}=0$. In all cases, we use $N=9$ and approximate the delta functions of (53) by square target reflectors of size $344 \times 344 \mathrm{~m}^{2}$, each having a unit reflectivity $\left(\mu_{n}=1\right)$. These reflectors are located in a scene of size $[0,22] \times[0,22] \mathrm{km}^{2}$ with flat topography. We discretize the scene by $128 \times 128$ pixels. Figs. 5 and 17 show the scene, receiver trajectories and the transmit antenna locations. In all cases, we use isotropic receiver antennas.

We use a transmitted pulse at center frequency $0 \mathrm{~Hz}$ with bandwidth equal to $.873 \mathrm{MHz}$ in computing the projection data. We heuristically chose the sampling rates of 1.746 and 670.25 MHz in fast-time and slow-time, respectively. At these rates, the area under the numerically computed point spread function of the imaging operator is small enough as compared to the size of the point scatterers in the scene that the resulting images are alias free.

For a collection of point scatterers, the normal direction of the edge is given by all directions (for a precise definition of a point scatterer and normal direction of an edge, see [38]). Therefore, a flight trajectory encircling the scatterers provides the best data collection manifold. Thus, for the single receiver case, we choose a circular flight trajectory $\boldsymbol{\gamma}_{C}(s)=(11+11 \cos (s), 11+$ $11 \sin (s), 6.5) \mathrm{km}$, uniformly sampled in $s \in[0,2 \pi)$ at 512 points (see Fig. 5), and consider isotropic stationary transmitter antennas.

For multiple receiver flight trajectories, we choose two receiver antennas traversing linear and parabolic flight trajectories, $\gamma_{L}(s)=(s, 0,6.5) \mathrm{km}$ and $\gamma_{P}(s)=$ $\left(\left(22 s-s^{2}\right) 22 / 121, s, 6.5\right) \mathrm{km}$, respectively, uniformly sampled for $s \in[0,22)$ at 512 points (see Fig. 17), and consider isotropic static noncooperative transmitter antennas.

For the single receiver and multiple transmitter case, we also include a numerical simulation in which the ground reflectivity is statistical. In target model (53), we assume that the $\left\{g_{n}\right\}$ are zero-mean normal random variables with variance $\sigma_{n}^{2}=1$. The projection data used in the reconstruction are generated from multiple realizations of the ground reflectivity.

For the single receiver cases, since $i=j=1$, for notational brevity, we drop the $i j(=11)$ dependence, i.e., we set $d_{11}\left(t, s, s^{\prime}\right)=d\left(t, s, s^{\prime}\right), r_{11}\left(s, s^{\prime}, \mathbf{x}\right)=r\left(s, s^{\prime}, \mathbf{x}\right), \boldsymbol{\xi}_{11}=\boldsymbol{\xi}$, etc.

\section{A. Single Transmitter and Single Receiver}

We consider the geometry shown in Fig. 5, with a receiver traversing the circular trajectory $\gamma(s)=\gamma_{C}(s)=$ $(11+11 \cos (s), 11+11 \sin (s), 6.5) \mathrm{km}$ (uniformly sampled for $s \in[0,2 \pi)$ at 512 points) and a stationary transmitter located at $\mathbf{y}_{0}=(0,0,6.5) \mathrm{km}$. The transmitter radiates a delta-like impulse. We generate the data [see Fig. 6(b)] by substituting $A_{R_{i}}(\omega, s, \boldsymbol{x})=|\boldsymbol{\gamma}(s)-\mathbf{x}|^{-1}$ and $A_{T}(\omega, \boldsymbol{x}, \mathbf{y})=\delta\left(\mathbf{y}-\mathbf{y}_{0}\right)|\mathbf{x}-\mathbf{y}|^{-1}$ in (1)

$$
\begin{aligned}
& d_{\mathbf{y}_{0}}(t, s) \\
& =\int \frac{\delta\left(t-\left[\left|\mathbf{y}_{0}-\mathbf{x}\right|+|\boldsymbol{\gamma}(s)-\mathbf{x}|\right] / c_{0}\right)}{|\boldsymbol{\gamma}(s)-\mathbf{x}|\left|\mathbf{x}-\mathbf{y}_{0}\right|} G(\boldsymbol{x}) d \boldsymbol{x} .
\end{aligned}
$$




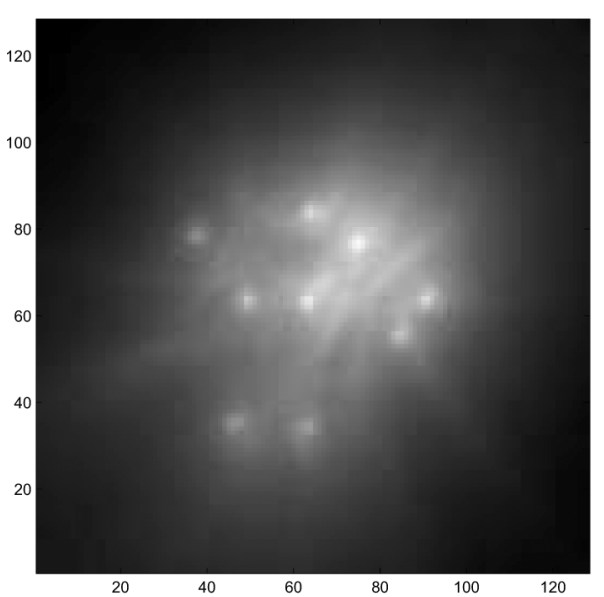

(a)

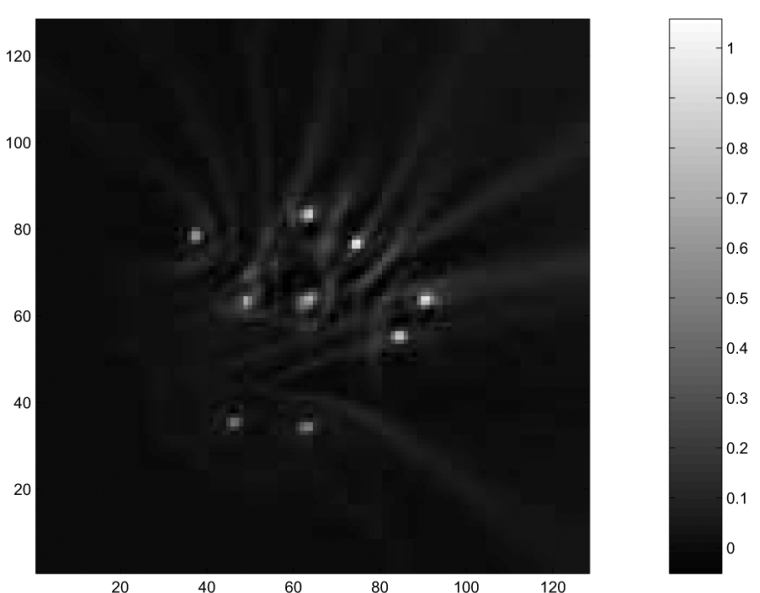

(b)

Fig. 7. Reconstructed images for SAH using (a) C-BP and (b) C-FBP for a single cooperative transmitter located at $\mathbf{y}_{0}$ and receiver traversing circular flight trajectory $\boldsymbol{\gamma}_{C}(s)$ (see Fig. 5 for explicit formula of $\gamma_{C}(s)$ ).

1) Cooperative Transmitter: If the transmitter is cooperative, then the corresponding C-FBP reconstruction formula becomes

$$
\begin{aligned}
& \hat{R}_{G}(\boldsymbol{z}) \sim\left|\mathbf{z}-\mathbf{y}_{0}\right|^{2} \int \mathrm{e}^{\mathrm{i} 2 \pi \omega\left(t-r\left(s, s^{\prime}, \mathbf{z}\right) / c_{0}\right)} \\
& \quad \times|\boldsymbol{\gamma}(s)-\mathbf{z}|\left|\boldsymbol{\gamma}\left(s+s^{\prime}\right)-\mathbf{z}\right|\left|\Xi_{1} \dot{\Xi}_{2}-\Xi_{2} \dot{\Xi}_{1}\right| \\
& \quad \times|\omega| d\left(t, s, s^{\prime}\right) d t d \omega d s d s^{\prime}
\end{aligned}
$$

where $\boldsymbol{\Xi}=\left(\Xi_{1}, \Xi_{2}\right)$, which by (36), is given by

$$
\begin{aligned}
\boldsymbol{\Xi}\left(s, s^{\prime}, \boldsymbol{z}, \boldsymbol{z}\right)=\left[\begin{array}{lll}
1 & 0 & 0 \\
0 & 1 & 0
\end{array}\right] \\
\\
\cdot\left[\frac{\mathbf{z}-\gamma(s)}{|\mathbf{z}-\gamma(s)|}-\frac{\mathbf{z}-\boldsymbol{\gamma}\left(s+s^{\prime}\right)}{\left|\mathbf{z}-\boldsymbol{\gamma}\left(s+s^{\prime}\right)\right|}\right] .
\end{aligned}
$$

For comparison purposes, we also perform C-BP reconstruction. The computational complexity of our implementation of C-FBP and C-BP are $\mathcal{O}\left(N^{4}\right)$ and $\mathcal{O}\left(N^{3} \log N\right)$, respectively. The corresponding reconstructed images are presented in Fig. 7. Our numerical simulations demonstrate that both the C-BP and C-FBP reconstructions produce all singularities at the correct location and orientation. This is due to the fact that the set $\Omega_{\mathrm{z}}$ contains all directions. The C-BP reconstruction appears as a smoothed version of the C-FBP one; this is because it is missing the ramp part of the filter (47). In general C-BP does not correctly capture the strength of the singularities, whereas C-FBP does as predicted by the theory.

Data Collection Manifold: Since visibility of an edge at $z$ is determined by the directions contained in the data collection manifold $\Omega_{\boldsymbol{z}}$, it is sufficient to consider the $\boldsymbol{\Xi}_{i j}\left(s, s^{\prime}, \boldsymbol{z}, \boldsymbol{z}\right)$ that are contained in the data collection manifold. Fig. 8 shows an illustration of the vectors $\boldsymbol{\Xi}\left(s, s^{\prime}, \boldsymbol{z}, \boldsymbol{z}\right)$ contained in the data collection manifolds $\Omega_{z, s^{\prime}=\pi / 4}$ and $\Omega_{\boldsymbol{z}}=\bigcup_{s^{\prime}} \Omega_{\boldsymbol{z}, s^{\prime}}$, respectively at various points $z$ in the scene. All the directions are included in both $\Omega_{z, s^{\prime}}$ and $\Omega_{z}$, however, $\Omega_{z, s^{\prime}}$ is significantly smaller than $\Omega_{z}$. This means that the spatial frequency content provided by
$\Omega_{z, s^{\prime}=\pi / 4}$ is less than that of $\Omega_{\boldsymbol{z}}$. To illustrate this numerically, we define

$$
\begin{aligned}
\hat{R}_{G_{\left(s^{\prime}=\pi / 4\right)}}(\boldsymbol{z}) & \mathrm{e}^{\mathrm{i} 2 \pi \omega\left(t-r\left(s, s^{\prime}=\pi / 4, \mathbf{z}\right) / c_{0}\right)} \\
= & \int_{\boldsymbol{x} i\left(\omega, s, s^{\prime}=\pi / 4, \boldsymbol{z}\right) \in \Omega_{\boldsymbol{z}, s^{\prime}=\pi / 4}} \\
& \times Q(\boldsymbol{z}, \omega, s, 0) d(s, 0, t) d t d \omega d s f
\end{aligned}
$$

and present $\hat{R}_{G_{\left(s^{\prime}=\pi / 4\right)}}$ in Fig. 9. Clearly, since $\Omega_{z, s^{\prime}=\pi / 4} \subset \Omega_{\boldsymbol{z}}$, the frequency content of $\hat{R}_{G_{\left(s^{\prime}=\pi / 4\right)}}$ is significantly less than $\hat{R}_{G}$ as shown in Figs. 9 and 7(b).

2) Noncooperative Transmitters: In the case of noncooperative transmitters, we assume that the scene is homogeneously illuminated by isotropic transmitters and set $\tilde{R}_{T}=1$. Consequently, we use the following reconstruction formula:

$$
\begin{aligned}
\hat{R}_{G}(z) \sim \int & \mathrm{e}^{\mathrm{i} 2 \pi \omega\left(t-r\left(s, s^{\prime}, \mathbf{z}\right) / c_{0}\right)}|\gamma(s)-\mathbf{z}|\left|\gamma\left(s+s^{\prime}\right)-\mathbf{z}\right| \\
& \times\left|\Xi_{1} \dot{\Xi}_{2}-\Xi_{2} \dot{\Xi}_{1}\right||\omega| d\left(t, s, s^{\prime}\right) d t d \omega d s d s^{\prime}
\end{aligned}
$$

Note that setting $\tilde{R}_{T}=1$ can be interpreted as an uninformative prior on the transmitter antenna beam patterns.

The reconstructed image for the case of a single noncooperative transmitter is presented in Fig. 10. With the loss of transmitter-related geometrical spreading factors in the reconstruction formula, the scatterers closer to the transmitter appear brighter than those that are further away. In the next subsection, we will see how this result may change with the introduction of additional transmitters illuminating the scene.

\section{B. Multiple Transmitters and Single Receiver}

In order to use MF-FBP [34] in the presence of multiple transmitters, we need to have the capability to isolate $d_{i, \mathbf{y}}$ from $d_{i}$ for each receiver location. This is not an easy task and sometimes not possible, especially in the presence of noncooperative sources of opportunity. Decomposition of the received signal, however, is not required when using C-FBP. 


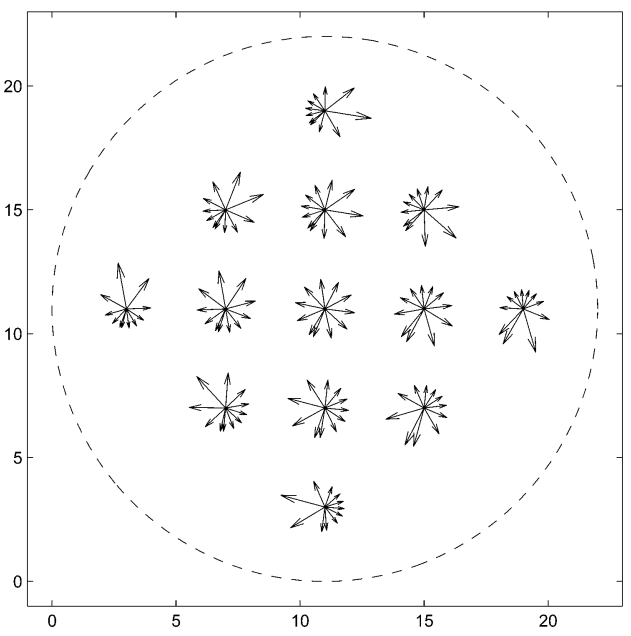

(a)

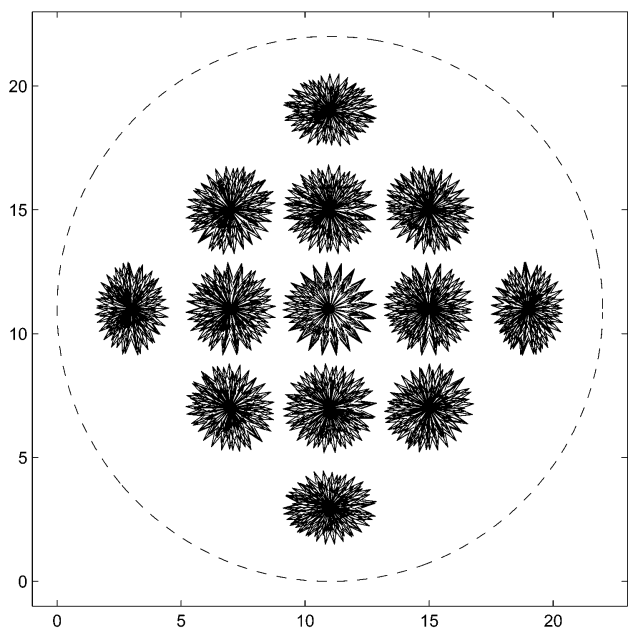

(b)

Fig. 8. Vectors $\boldsymbol{\Xi}\left(s, s^{\prime}, \boldsymbol{z}, \boldsymbol{z}\right)$ (see (56) and (34)) contained in the data collection manifolds (a) $\Omega_{\boldsymbol{z}, s^{\prime}}$ for the slow-time delay $s^{\prime}=\pi / 4$, and (b) $\Omega_{\boldsymbol{z}}=\bigcup_{s^{\prime}} \Omega_{\boldsymbol{z}, s^{\prime}}$ at various points $z$ within the target scene. The receiver is traversing $\gamma_{C}(s)$ (dashed line) (see Fig. 5 for the explicit formula of $\gamma_{C}(s)$ and Fig. 4 for an illustration of $\left.\boldsymbol{\Xi}\left(s, s^{\prime}, \boldsymbol{z}, \boldsymbol{z}\right)\right)$. Clearly, correlation of the received data at multiple viewing angles provides a larger data collection manifold, then the one with a single slow-time delay correlation.

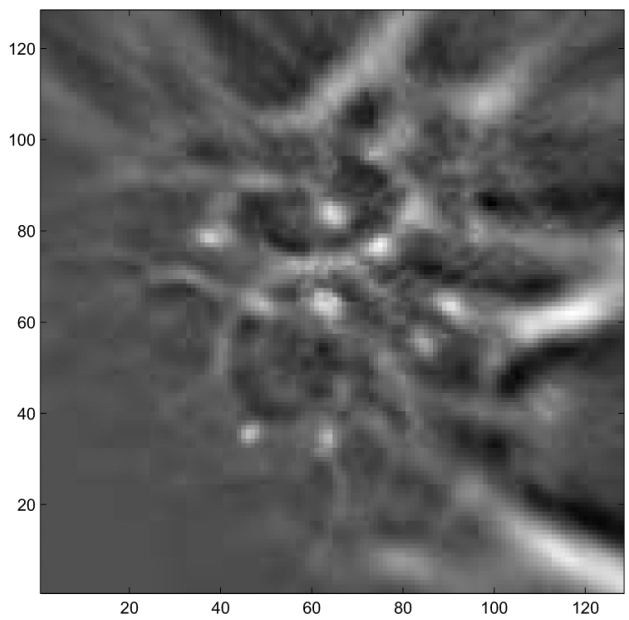

Fig. 9. $\hat{R}_{G_{\left(s^{\prime}=\pi / 4\right)}}$, the image reconstructed using the vectors in the data collection manifold $\Omega_{\boldsymbol{z}, s^{\prime}=\pi / 4}$ for a single cooperative transmitter located at $\mathbf{y}_{0}$ as shown in Fig. 5(a) and a receiver traversing a circular flight trajectory $\gamma_{C}(s)$ (see Fig. 5 for an explicit formula of $\gamma_{C}(s)$ ). Note that $\Omega_{z, s^{\prime}=\pi / 4}$ is formed by correlating the received signal at a single slow-time delay, $s^{\prime}=p i / 4$. This reconstruction when compared to the one in Fig. 7(b) shows that correlating the received data at multiple slow-time delays on the flight trajectory improves the reconstructed image as predicted by the theory.

We demonstrate the performance of the C-FBP method in the presence of multiple transmitters in two sets of numerical simulations. In the first set of simulations, we consider two isotropic transmitters at $\mathbf{y}_{0}=(0,0,6.5) \mathrm{km}$ and $\mathbf{y}_{1}=(22,22,6.5)$ $\mathrm{km}$. In the second set of simulations, we consider four isotropic transmitters, located at $\mathbf{y}_{0}, \mathbf{y}_{1}, \mathbf{y}_{2}=(0,22,6.5) \mathrm{km}$, and $\mathbf{y}_{3}=$ $(22,0,6.5) \mathrm{km}$. In both cases, the receiver follows the path $\boldsymbol{\gamma}_{C}$ as before. We generated data $d$ by adding up the separately generated data for each of the transmitters by

$$
d(t, s)=\sum_{i} d_{\mathbf{y}_{i}}(t, s) .
$$

We show data for the two- and four-transmitter cases in Fig. 11.

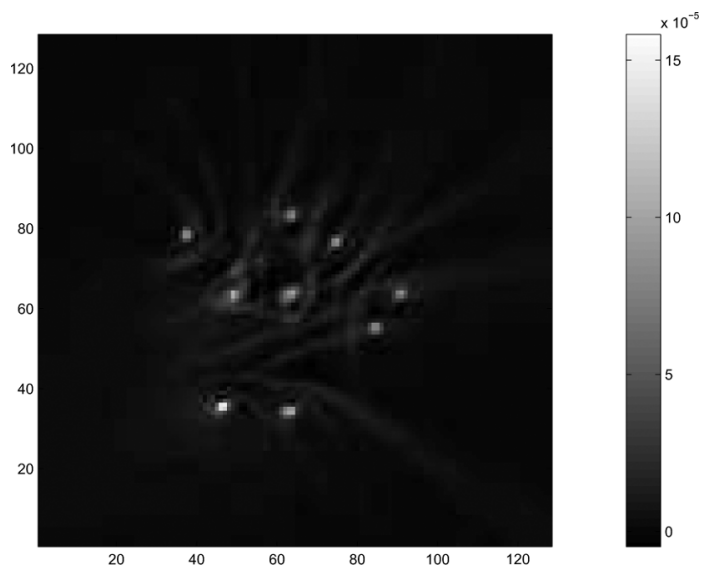

Fig. 10. Reconstructed image by C-FBP using (58) for a single noncooperative transmitter located at $\mathbf{y}_{0}$ and receiver traversing the circular flight trajectory $\gamma_{C}(s)$ (see Fig. 5 for explicit formula of $\gamma_{C}(s)$ ).

1) Cooperative Transmitters: In the case of cooperative transmitters, we have

$$
A_{T}(\omega, \boldsymbol{x}, \mathbf{y})=\frac{\sum_{i} \delta\left(\mathbf{y}-\mathbf{y}_{i}\right)}{|\mathbf{x}-\mathbf{y}|}
$$

therefore

$$
\begin{aligned}
\tilde{R}_{T}(\omega, \boldsymbol{x}) \\
=\left[\int \mathrm{e}^{\left.\mathrm{i} 2 \pi \omega|\mathbf{y}-\mathbf{x}| / c_{0} \frac{\sum_{i} \delta\left(\mathbf{y}-\mathbf{y}_{i}\right)}{|\mathbf{x}-\mathbf{y}|} d \mathbf{y}\right]}\right. \\
\quad \times\left[\int \mathrm{e}^{\left.\mathrm{i} 2 \pi \omega\left|\mathbf{y}^{\prime}-\mathbf{x}\right| / c_{0} \frac{\sum_{j} \delta\left(\mathbf{y}^{\prime}-\mathbf{y}_{j}\right)}{\left|\mathbf{x}-\mathbf{y}^{\prime}\right|} d \mathbf{y}^{\prime}\right]^{*}}\right. \\
=\sum_{i, j} \mathrm{e}^{\mathrm{i} 2 \pi \omega\left(\left|\mathbf{y}_{i}-\mathbf{x}\right|-\left|\mathbf{y}_{j}-\mathbf{x}\right|\right) / c_{0}} \frac{1}{\left|\mathbf{x}-\mathbf{y}_{i}\right|\left|\mathbf{x}-\mathbf{y}_{j}\right|} \\
=\sum_{i, j>i}\left[\frac{1}{\left|\mathbf{x}-\mathbf{y}_{i}\right|^{2}}+\frac{\cos \left(2 \pi \omega\left[\left|\mathbf{y}_{i}-\mathbf{x}\right|-\left|\mathbf{y}_{j}-\mathbf{x}\right|\right]\right)}{\left|\mathbf{x}-\mathbf{y}_{i}\right|\left|\mathbf{x}-\mathbf{y}_{j}\right|}\right] .
\end{aligned}
$$




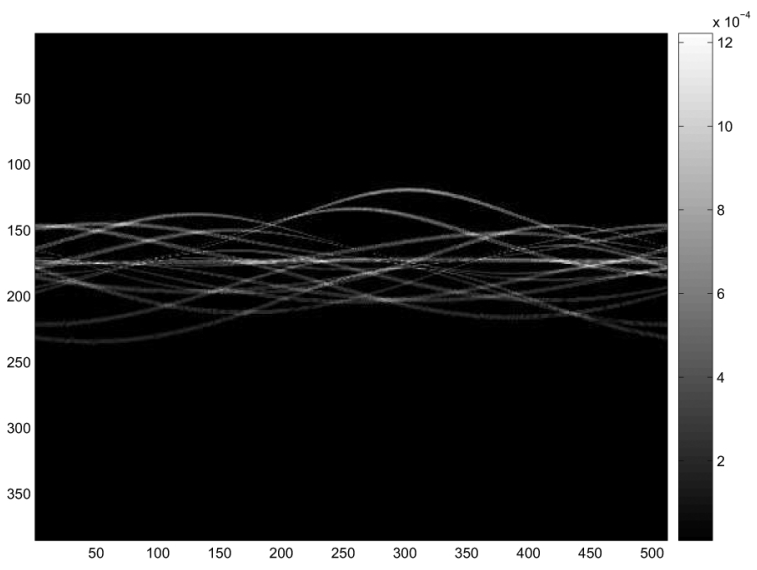

(a)

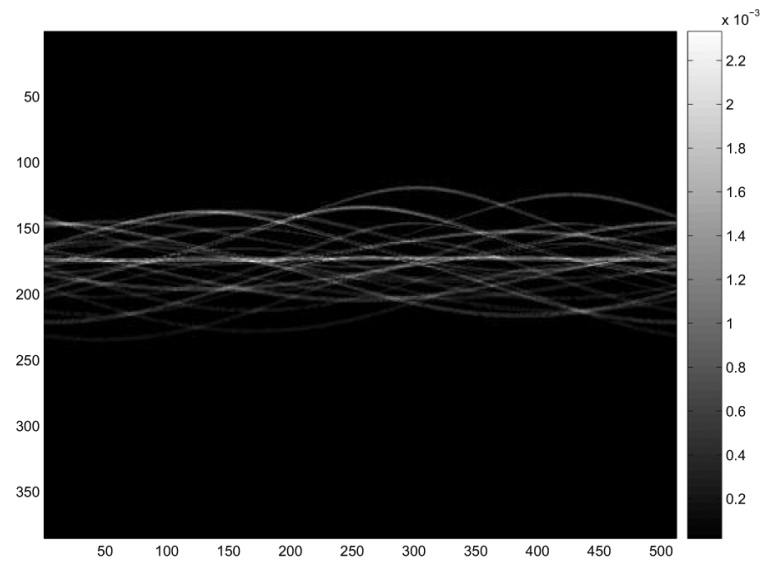

(b)

Fig. 11. Data collected over a circular receiver trajectory $\boldsymbol{\gamma}_{C}(s)$ due to (a) two transmitters located at $\mathbf{y}_{0}$, and $\mathbf{y}_{1}$ and (b) four transmitters located at $\mathbf{y}_{0}, \mathbf{y}_{1}, \mathbf{y}_{2}$, and $\mathbf{y}_{3}$ (see Fig. 5 for explicit formula of $\gamma_{C}(s)$ ). Note that grey scales are different for the two plots; the data is larger in magnitude for the four-transmitter case, as expected.

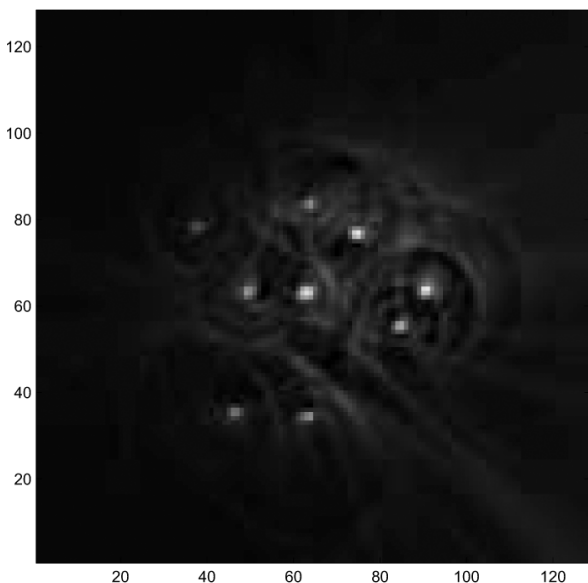

(a)
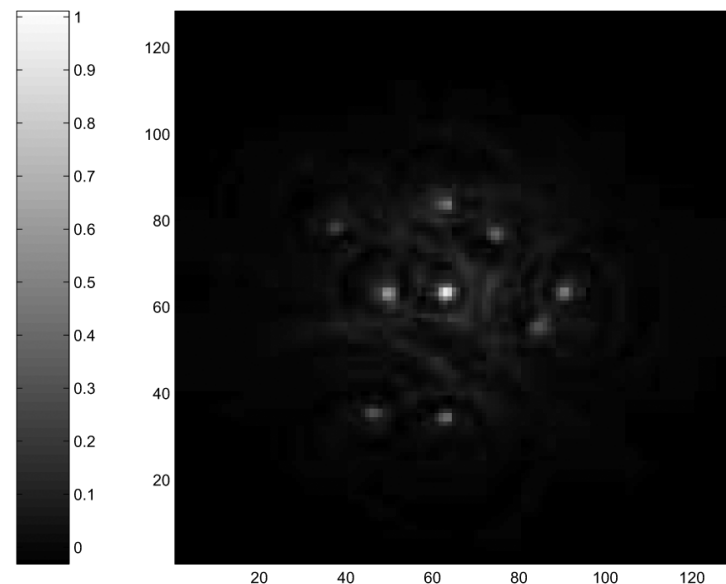

(b)

Fig. 12. Reconstructed images using C-FBP in the presence of (a) two cooperative transmitters located at $\mathbf{y}_{0}$ and $\mathbf{y}_{1}$, and (b) four cooperative transmitters located at $\mathbf{y}_{0}, \mathbf{y}_{1}, \mathbf{y}_{2}$ and $\mathbf{y}_{3}$. In both cases, the receiver traverses the circular trajectory $\gamma_{C}(s)$ (see Fig. 5 for explicit formula of $\gamma_{C}(s)$ ).

Since transmitters are far away from the scene, lie at the same altitude, and are equidistant from the center of the scene, we make the approximation $\left|\mathbf{x}-\mathbf{y}_{\boldsymbol{i}}\right| \approx\left|\mathbf{x}-\mathbf{y}_{j}\right|$ in (61), and write

$$
\tilde{R}_{T}(\omega, x) \approx \sum_{i, j>i}\left[\frac{1}{\left|\mathbf{x}-\mathbf{y}_{i}\right|^{2}}+\frac{1}{\left|\mathbf{x}-\mathbf{y}_{i}\right|\left|\mathbf{x}-\mathbf{y}_{j}\right|}\right]
$$

Replacing $\tilde{R}_{T}$ in $A_{11}$ with (62), the C-FBP formula becomes

$$
\begin{aligned}
\hat{R}_{G}(z)=\left(\sum_{i, j>i}\left[\frac{1}{\left|\mathbf{z}-\mathbf{y}_{i}\right|^{2}}+\frac{1}{\left|\mathbf{z}-\mathbf{y}_{i}\right|\left|\mathbf{z}-\mathbf{y}_{j}\right|}\right]\right)^{-1} \\
\times \hat{R}_{G}(z)
\end{aligned}
$$

where $\hat{R}_{G}$ is the reconstruction for noncooperative transmitters given in (58).

We present the reconstructed images using C-FBP for twoand four-cooperative transmitter cases in Fig. 12. For comparison purposes, we also present the $\mathrm{C}$ - $\mathrm{BP}$ reconstruction for the two- and four-cooperative transmitter case in Fig. 13.

Reconstructed images show that the C-FBP method for two and four transmitters produces images that are similar to the image reconstructed in the presence of single transmitter presented in Fig. 7. Similar to the single transmitter case, both C-BP and C-FBP methods lead to reconstruction of singularities at the right location and orientation in the presence of both cooperative and noncooperative sources of opportunity. Furthermore, in the presence of cooperative sources of opportunity, the singularities reconstructed by C-FBP have the correct strength, as suggested by the theory.

2) Noncooperative Transmitters: If the transmitters are noncooperative, we perform the C-FBP reconstruction by using (58). We present the reconstructed images for two and four noncooperative transmitters in Fig. 14. The reconstructed images show that while the singularities appear at the correct location, they do not necessarily have the correct strength.

Note that the performance of the C-BP is the same for both cooperative and noncooperative sources since the transmitterrelated information is needed only at the filtering step which is not present in the C-BP algorithm.

3) Single Receiver and Multiple Transmitters With Statistical Ground Reflectivity: We consider the scene radiance (53) in which the means $\mu_{n}$ are all zero.

We generate the expected data $d$ by averaging the data obtained from ten different realizations of the scene reflectivity (52). Fig. 15 shows the expected value of the data for four transmitters located at $\mathbf{y}_{0}, \mathbf{y}_{1}, \mathbf{y}_{2}$ and $\mathbf{y}_{3}$ as shown in Fig. 5. 


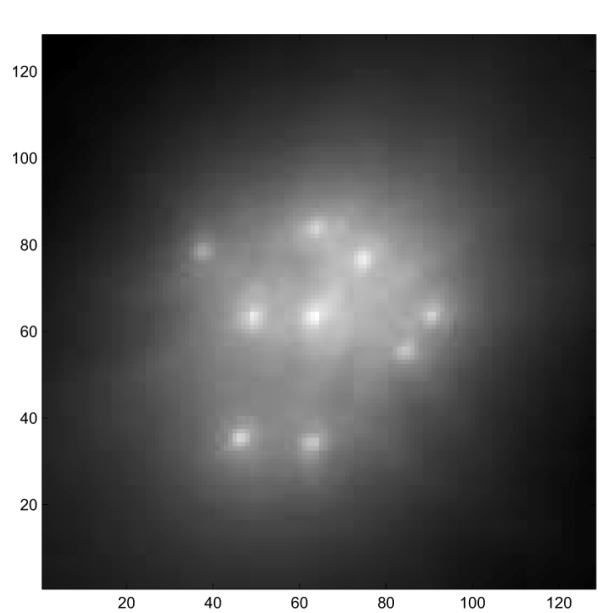

(a)
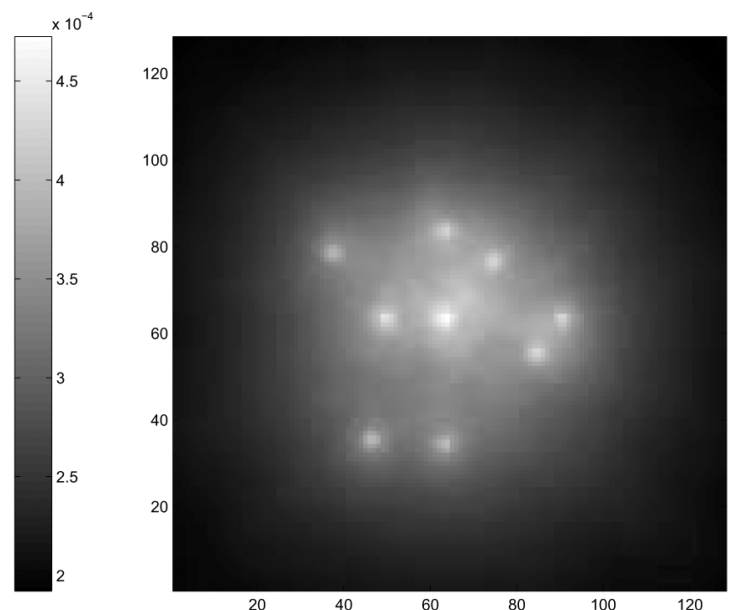

(b)

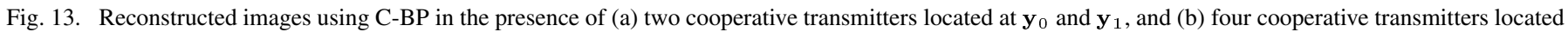
at $\mathbf{y}_{0}, \mathbf{y}_{1}, \mathbf{y}_{2}$, and $\mathbf{y}_{3}$. In both cases, the receiver traverses the circular trajectory $\boldsymbol{\gamma}_{C}(s)$ (see Fig. 5 for explicit formula of $\gamma_{C}(s)$ ).

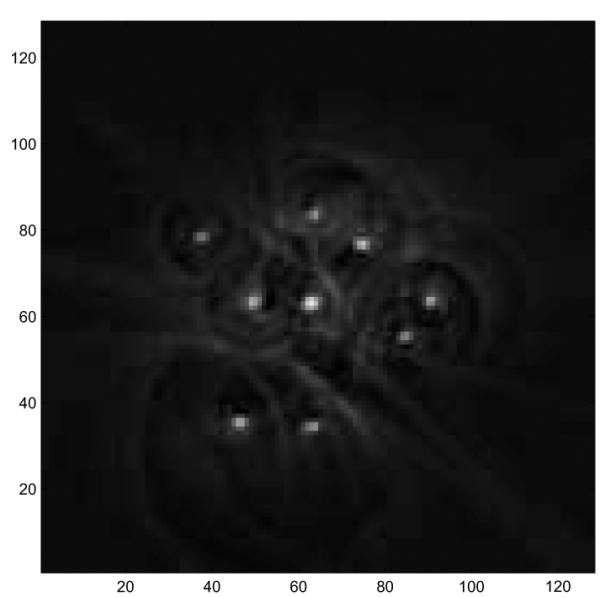

(a)
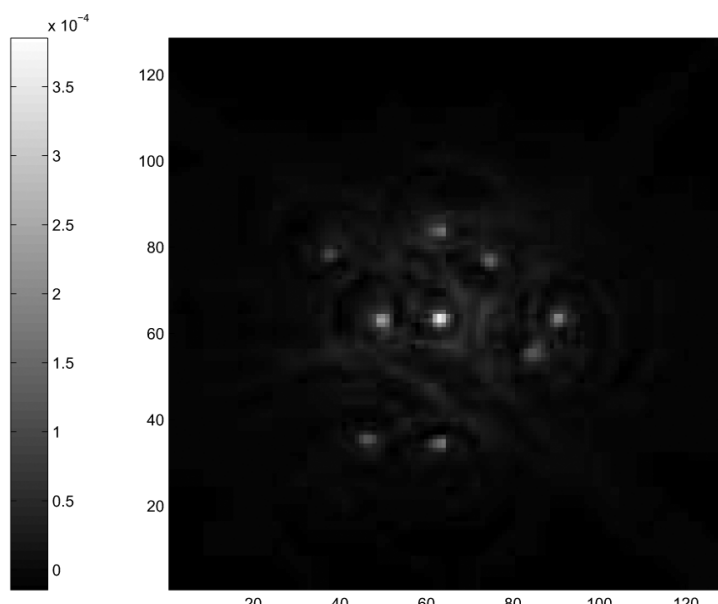

(b)

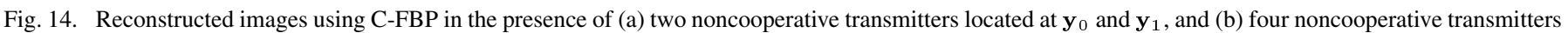
located at $\mathbf{y}_{0}, \mathbf{y}_{1}, \mathbf{y}_{2}$, and $\mathbf{y}_{3}$. In both cases, the receiver traverses the circular trajectory $\boldsymbol{\gamma}_{C}(s)$ (see Fig. 5 for explicit formula of $\gamma_{C}(s)$ ).

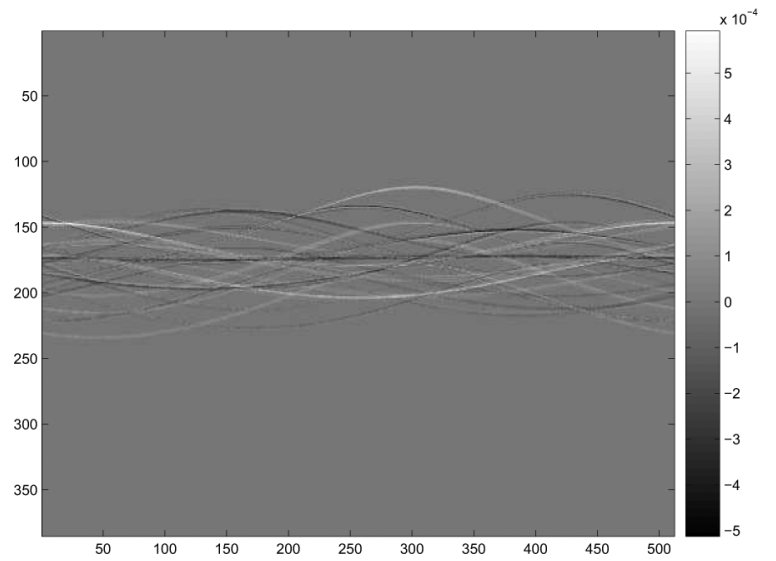

Fig. 15. Expected projection data collected over a circular receiver trajectory $\boldsymbol{\gamma}_{C}$ due to transmitters located at $\mathbf{y}_{0}, \mathbf{y}_{1}, \mathbf{y}_{2}$ and $\mathbf{y}_{3}$ (see Fig. 5 for explicit formula of $\left.\gamma_{C}(s)\right)$ for a statistical reflectivity function.

The corresponding reconstructed radiance image using (58) in the presence of noncooperative transmitters and using (63) in the presence of cooperative transmitters are presented in Fig. 16. Note that for both the cooperative and noncooperative cases, the reconstructed image for the statistical ground reflectivity has fewer artifacts than the deterministic reconstruction. This can be explained by the fact that the scene radiance satisfies assumption (8), and reconstruction uses data averaged averaged over several passes, which has a better compliance with the assumptions of our method as shown in (12) and (17).

This can explained by the fact that the scene radiance satisfies assumption (8).

\section{Multiple Receivers and Single Transmitter}

In the previous two subsections, we presented and compared the performance of C-BP and C-FBP reconstruction methods for a single receiver trajectory in the presence of both single and multiple cooperative and noncooperative sources of opportunity. In this and the following subsection, we demonstrate the performance of our method for multiple airborne receivers. For brevity, we consider only noncooperative sources of opportunity. 


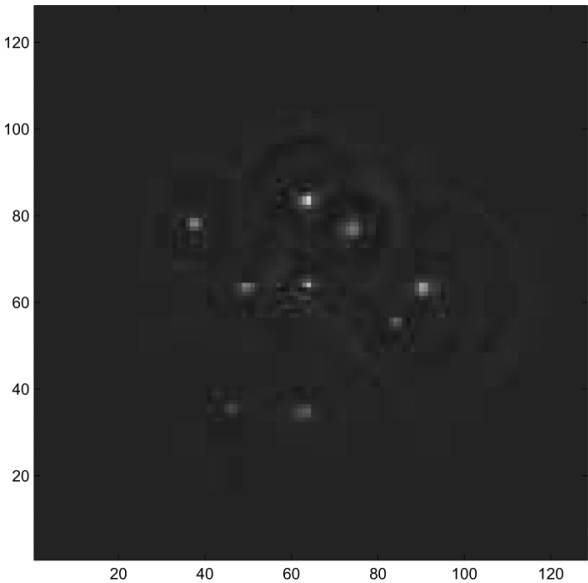

(a)

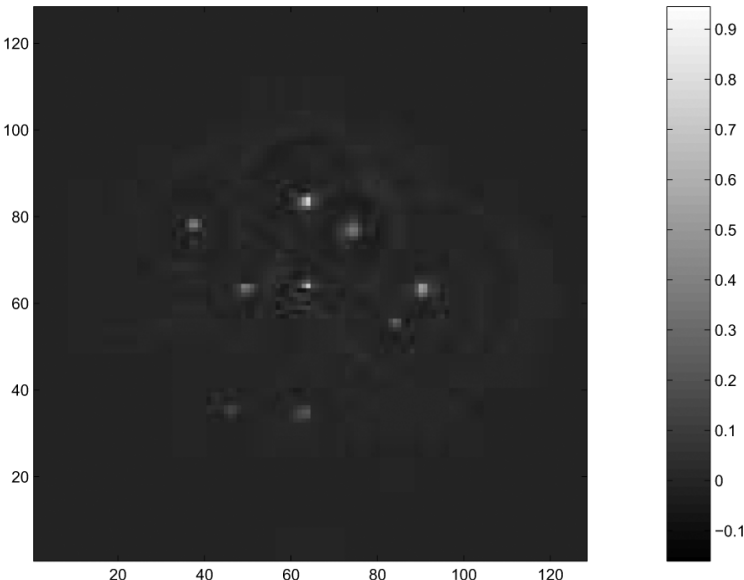

(b)

Fig. 16. Reconstructed images for the statistical ground reflectivity using C-FBP in the presence of (a) noncooperative and (b) cooperative transmitters located at $\mathbf{y}_{0}, \mathbf{y}_{1}, \mathbf{y}_{2}$, and $\mathbf{y}_{3}$ and receiver traversing circular trajectory $\boldsymbol{\gamma}_{C}(s)$ (see Fig. 5 for explicit formula of $\boldsymbol{\gamma}_{C}(s)$ ).

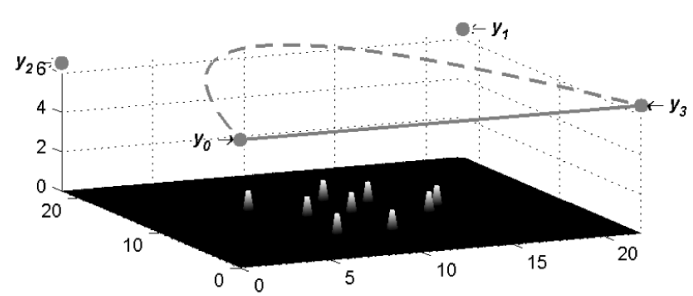

(a)

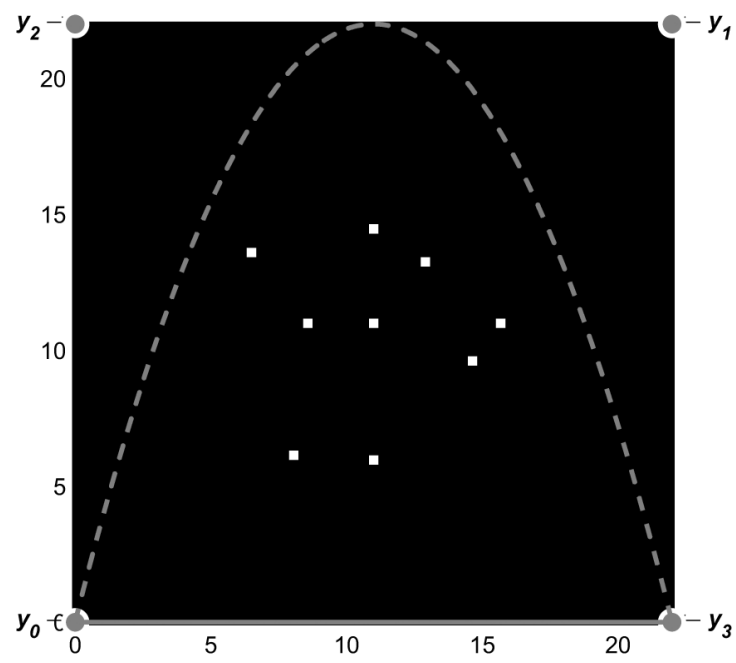

(b)

Fig. 17. (a) 3-D and (b) 2-D views of the scene with transmitters located at $\mathbf{y}_{0}=(0,0,6.5) \mathrm{km}, \mathbf{y}_{1}=(22,22,6.5) \mathrm{km}, \mathbf{y}_{2}=(0,22,6.5) \mathrm{km}$, and $\mathbf{y}_{3}=(22,0,6.5) \mathrm{km}$, and linear (solid line), $\boldsymbol{\gamma}_{L}(s)=(s, 0,6.5) \mathrm{km}$, and parabolic (dashed line), $\gamma_{P}(s)=\left(22 s-s^{2}, s, 6.5\right) \mathrm{km}$, receiver trajectories.

We consider two receiver flight trajectories, namely, linear and parabolic: $\boldsymbol{\gamma}_{L}(s)=(s, 0,6.5) \mathrm{km}$ and $\gamma_{P}(s)=((22 s-$ $\left.\left.s^{2}\right) 22 / 121, s, 6.5\right) \mathrm{km}$, respectively, uniformly sampled for $s \in$ $[0,22)$ at 512 points.

We use a single noncooperative transmitter located at $\mathbf{y}_{0}=$ $(0,0,6.5) \mathrm{km}$, as shown in Fig. 17 , radiating a delta-like impulse. As in Section VI-A, we generated the data using (54).
The data for linear and parabolic receiver trajectories are presented in Fig. 18. The corresponding C-BP and C-FBP reconstructions using (58) are presented in Figs. 19 and 20, respectively.

The two reconstructed images capture different frequency content of the scene. In particular, let $\Omega_{i j, \boldsymbol{z}}=\bigcup_{s^{\prime}} \Omega_{i j, \boldsymbol{z}, s^{\prime}}$ denote the frequency content of the image obtained from the correlation of the data obtained from the $i$ th and $j$ th receiver trajectories, $i, j \in\{1,2\}$. The frequency content contribution $\Omega_{11, z}$ due to the linear receiver flight trajectory is significantly less than that of the parabolic receiver flight trajectory, $\Omega_{22, z}$ [see Fig. 21(a) and (b)]. This is a direct consequence of the fact that the linear flight trajectory provides a smaller aperture than that of the parabolic flight trajectory.

Summing the reconstructed images obtained from the linear and parabolic receiver flight union of $\Omega_{11, z}$ and $\Omega_{22, z}$ [ee Fig. 22(a) and (b)]. The frequency content of the reconstructed image can be further increased by incorporating the image reconstructed from the cross correlation of the data obtained from the linear and parabolic trajectories. A diagram of $\boldsymbol{\Xi}_{i j}\left(s, s^{\prime}, \boldsymbol{z}, \boldsymbol{z}\right), i, j \in\{1,2\}$, contained in the cross terms, $\Omega_{12, \boldsymbol{z}}=-\Omega_{21, \boldsymbol{z}}$, and in the data collection manifold $\Omega_{\boldsymbol{z}}=\sum_{i, j \in\{1,2\}} \Omega_{i j, \boldsymbol{z}}$, are shown in Fig. 21(c) and (d). The $\mathrm{C}-\mathrm{BP}$ and C-FBP reconstructed images with the frequency content $\Omega_{z}$ are presented in Fig. 22(c) and (d), respectively.

As in the previous cases, both $\mathrm{C}-\mathrm{BP}$ and $\mathrm{C}-\mathrm{FBP}$ reconstruct the singularities at the correct location and orientation. Furthermore, since C-FBP method corrects for the receiver-related amplitude factors, the amplitude of the singularities are better captured by C-FBP than by C-BP, as shown in Fig. 22.

\section{Multiple Receivers and Multiple Transmitters}

Finally, we consider four noncooperative transmitters located at $\mathbf{y}_{0}=(0,0,6.5) \mathrm{km}, \mathbf{y}_{1}=(22,22,6.5) \mathrm{km}, \mathbf{y}_{2}=(0,22,6.5)$ $\mathrm{km}$, and $\mathbf{y}_{3}=(22,0,6.5) \mathrm{km}$ and the two receiver flight trajectories, namely, linear $\left(\boldsymbol{\gamma}_{L}(s)\right)$ and parabolic $\left(\boldsymbol{\gamma}_{P}(s)\right)$ as shown in Fig. 17. 


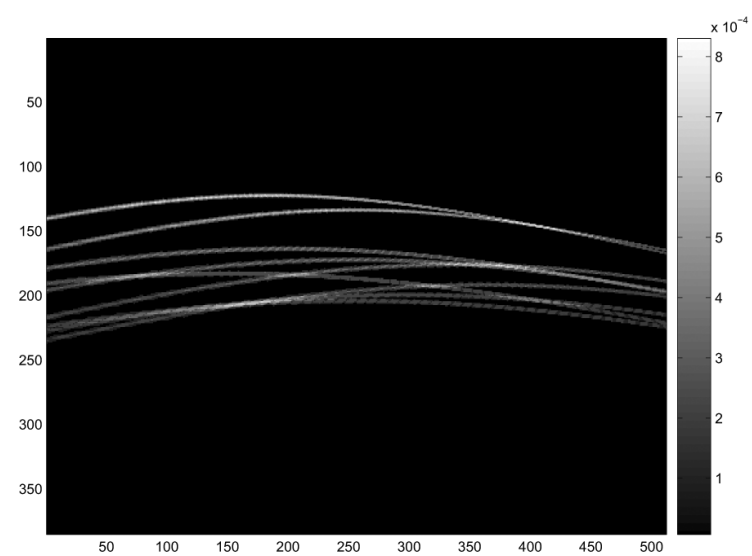

(a)

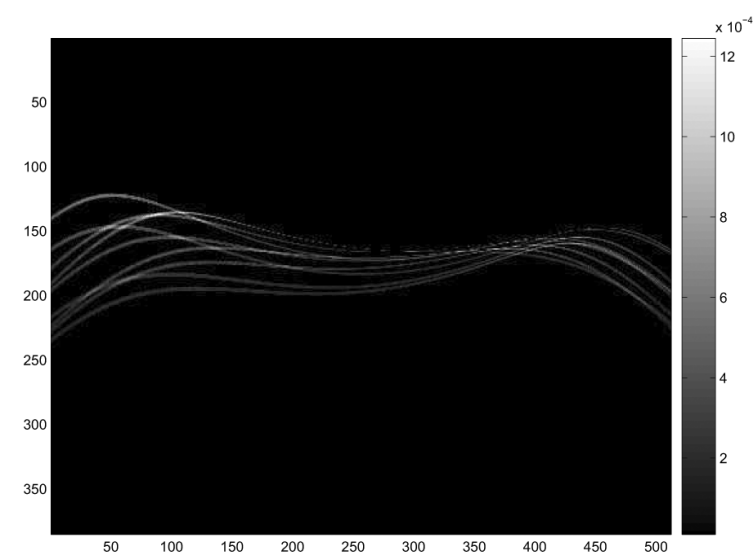

(b)

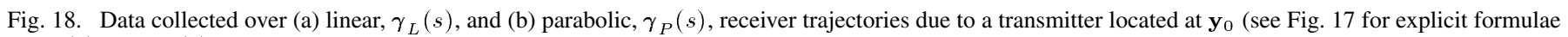
of $\gamma_{L}(s)$ and $\left.\gamma_{P}(s)\right)$.

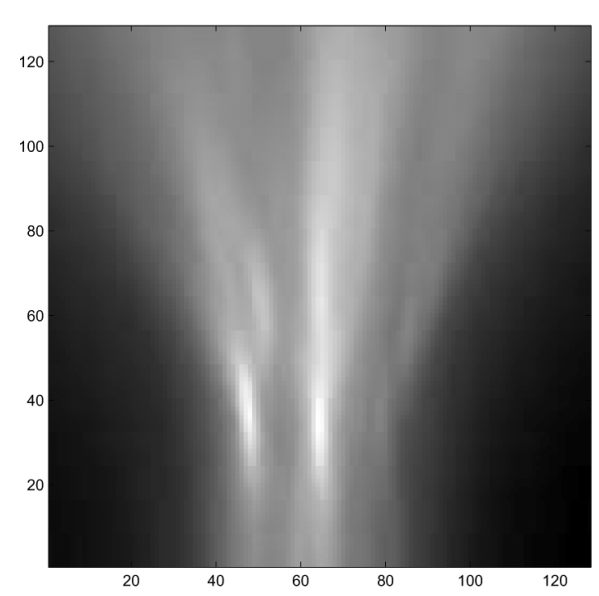

(a)
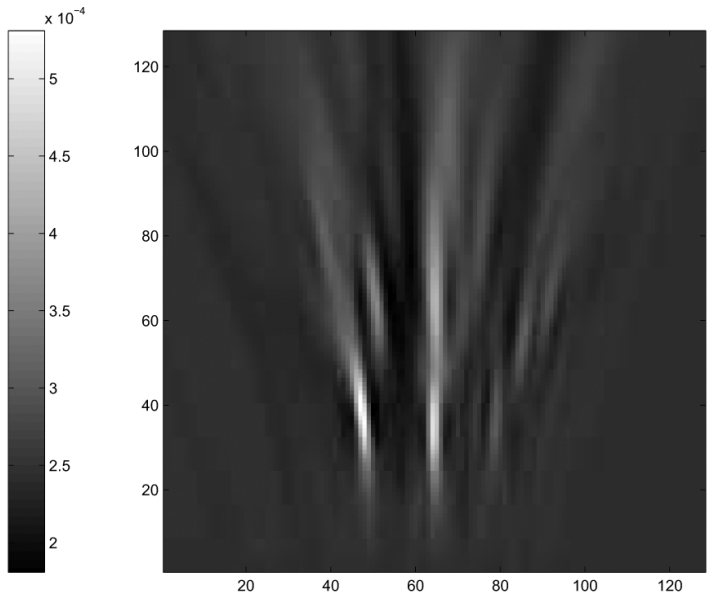

(b)

Fig. 19. Reconstructed images using (a) C-BP and (b) C-FBP for a single noncooperative transmitter located at $\mathbf{y}_{0}$ and a linear receiver trajectory, $\gamma_{L}(s)$ (see Fig. 17 for explicit formula of $\gamma_{L}(s)$ ).

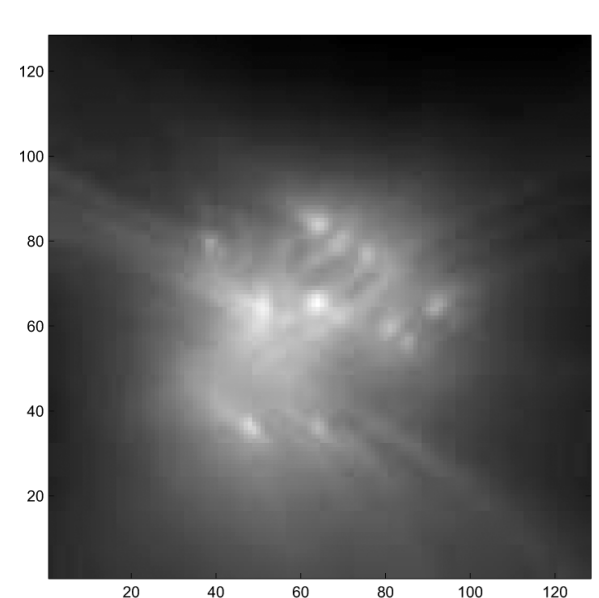

(a)
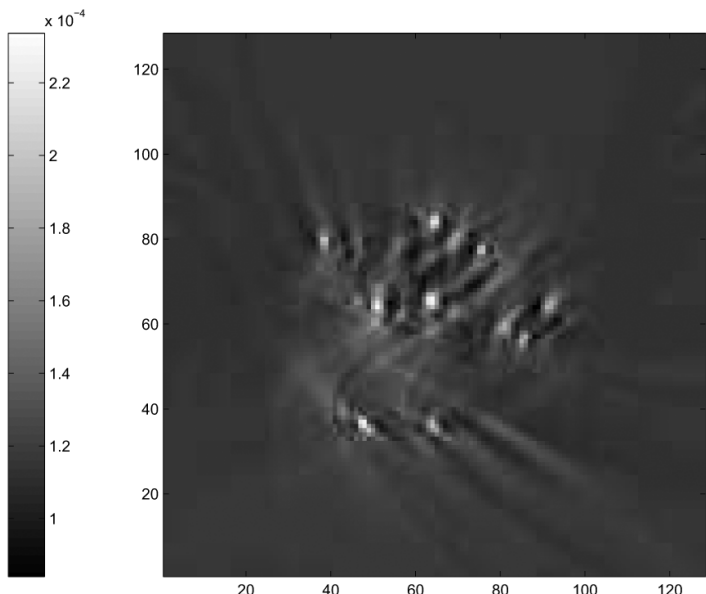

(b)

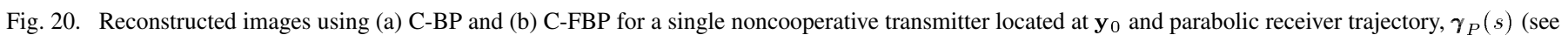
Fig. 17 for explicit formula of $\gamma_{P}(s)$ ).

The reconstructed C-BP and C-FBP images are shown in Fig. 23. As in the multiple-receiver, single-transmitter case, the datacollection manifold increases with the inclusion of the cross- correlation term. Compared to the single-transmitter case, the strengths of the singularities are improved in the four-transmitter case. 


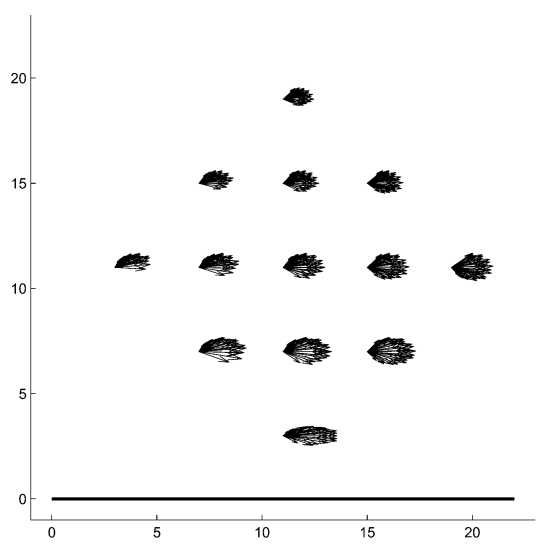

(a)

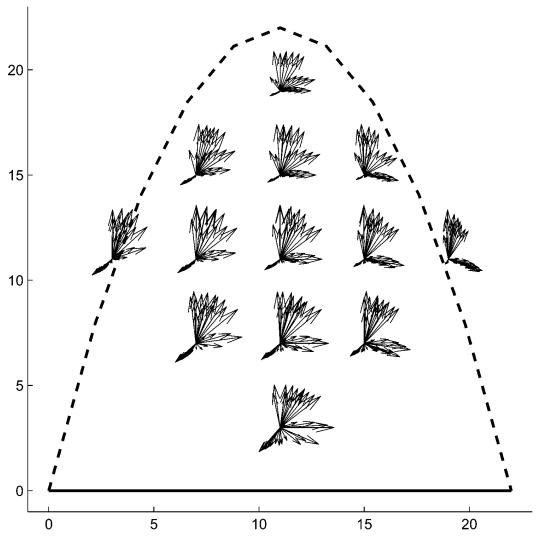

(c)

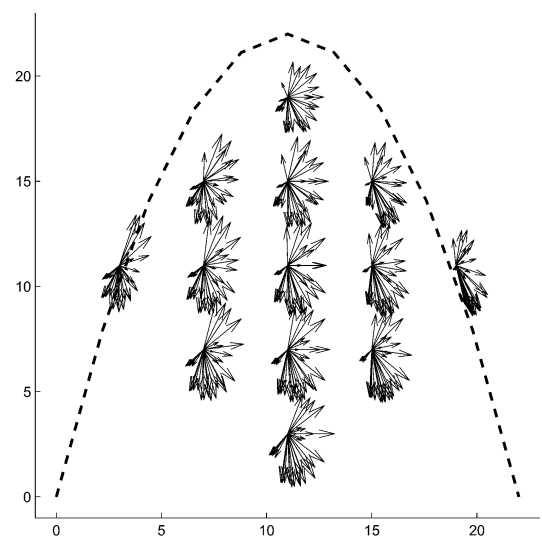

(b)

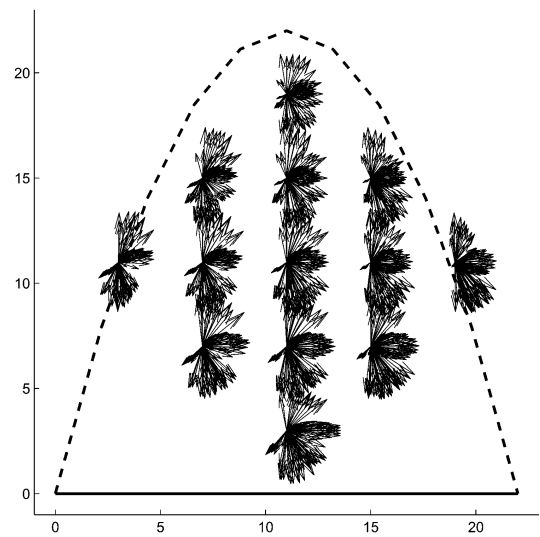

(d)

Fig. 21. Vectors $\boldsymbol{\Xi}_{i j}\left(s, s^{\prime}, \boldsymbol{z}, \boldsymbol{z}\right), i, j \in\{1,2\}$ (see (36)), contained in the data collection manifolds (a) $\Omega_{11, z}$ (obtained by autocorrelation of data collected over linear flight trajectory), (b) $\Omega_{22, z}$ (obtained by autocorrelation of data collected over parabolic flight trajectory), (c) $\Omega_{12, z}=-\Omega_{21, z}$ (obtained by crosscorrelation of data collected over linear and parabolic flight trajectories), and (d) $\Omega_{\boldsymbol{z}}=\bigcup_{i, j \in\{1,2\}} \Omega_{i j, z}$ at various points within the target scene. Here, the linear (solid line) and parabolic (dashed line) receiver trajectories are $\gamma_{1}(s)=\gamma_{L}(s)$ and $\gamma_{2}(s)=\gamma_{P}(s)$, respectively (see Fig. 17 for explicit formulae of $\boldsymbol{\gamma}_{L}(s)$ and $\left.\gamma_{P}(s)\right)$.

\section{CONCLUSION}

We presented a novel image reconstruction method, correlation-filtered-backprojection (C-FBP), for synthetic-aperture hitchhiker systems in the presence of cooperative and noncooperative sources of opportunity using single or multiple receivers.

We combined correlation-based imaging and microlocal techniques to provide an analytic inversion formula to recover ground radiance. The inversion formula has the desirable property of reconstructing the visible edges of the scene at the correct location and orientation and at the correct strength for the case of cooperative sources of opportunity.

We developed an algorithm to implement the C-FBP inversion formula and analyzed its computational complexity. We demonstrated the performance of the C-FBP algorithm in comparison to $\mathrm{C}-\mathrm{BP}$ algorithm in extensive simulations.

The method is also applicable to mobile sources of opportunity following arbitrary trajectories. The method can be extended to include the case where the projection data is contaminated with additive noise following the framework introduced in our prior work [38].

Additionally, the method can be also utilized in optical coherence tomography, passive/micro seismic imaging and other passive or active imaging modalities.

\section{APPENDIX}

The stationary phase theorem states [41]-[43] that if $a$ is a smooth function of compact support on $\mathbb{R}^{n}$, and $\phi$ has only nondegenerate critical points, then as $\omega \rightarrow \infty$

$$
\begin{aligned}
\int \mathrm{e}^{\mathrm{i} 2 \pi \omega \phi(\boldsymbol{x})} a(\boldsymbol{x}) d^{n} \boldsymbol{x} \\
=\sum_{\left\{\boldsymbol{x}^{0}: D \phi\left(\boldsymbol{x}^{0}\right)=0\right\}}\left(\frac{1}{\omega}\right)^{n / 2} a\left(\boldsymbol{x}^{0}\right) \\
\quad \times \frac{\mathrm{e}^{\mathrm{i} 2 \pi \omega \phi\left(\boldsymbol{x}^{0}\right)} e^{i(\pi / 4) \operatorname{sgn} D^{2} \phi\left(\boldsymbol{x}^{0}\right)}}{\sqrt{\left|\operatorname{det} D^{2} \phi\left(\boldsymbol{x}^{0}\right)\right|}} \\
\quad+O\left(\omega^{-n / 2-1}\right) .
\end{aligned}
$$

Here $D \phi$ denotes the gradient of $\phi, D^{2} \phi$ denotes the Hessian, and sgn denotes the signature of a matrix, i.e., the number of positive eigenvalues minus the number of negative ones.

\section{ACKNOWLEDGMENT}

The authors would like to thank M. Cheney for fruitful discussions. 


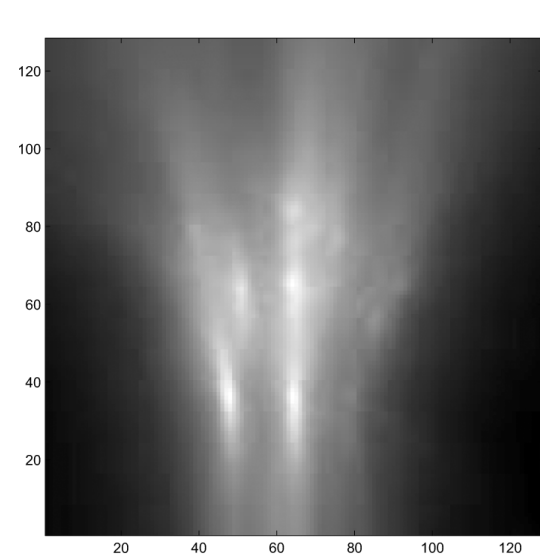

(a)

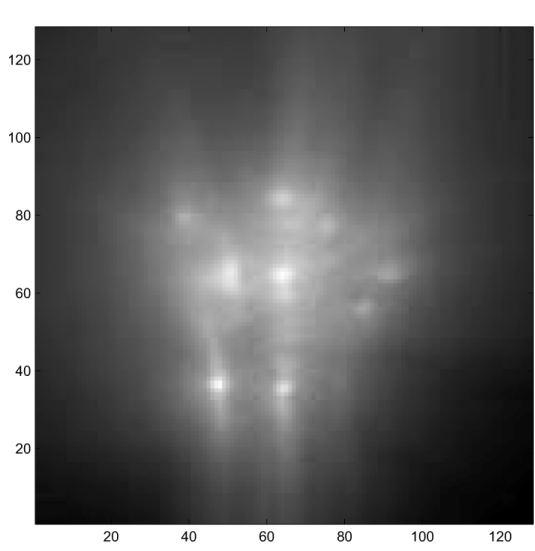

(c)
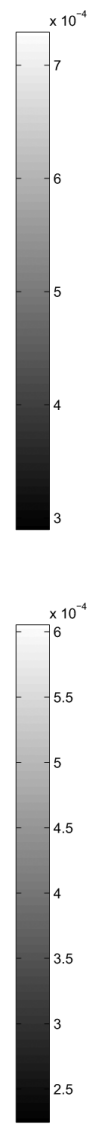

\section{(1)}

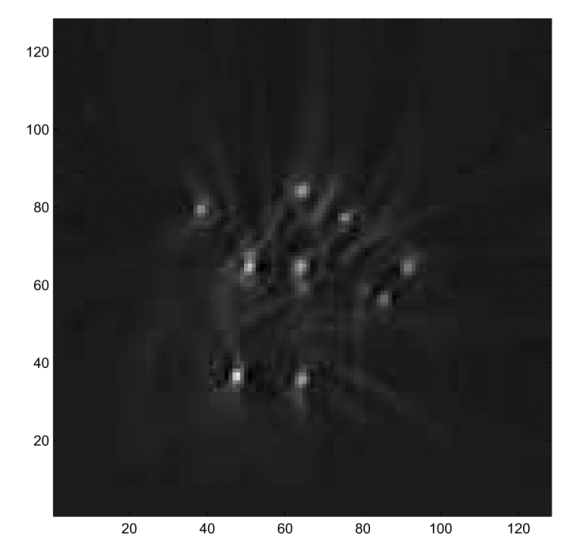

(d)

Fig. 22. Sum of the (a) C-BP and (b) C-FBP reconstructed images for linear and parabolic trajectories presented in Figs. 19 and 20 . Reconstructed images using (c) C-BP and (d) C-FBP for a single noncooperative transmitter located at $\mathbf{y}_{0}$ and receivers traversing linear and parabolic flight trajectories $\gamma_{L}(s)$ and $\gamma_{P}(s)$, respectively (see Fig. 17 for explicit formulae of $\gamma_{L}(s)$ and $\gamma_{P}(s)$ ).

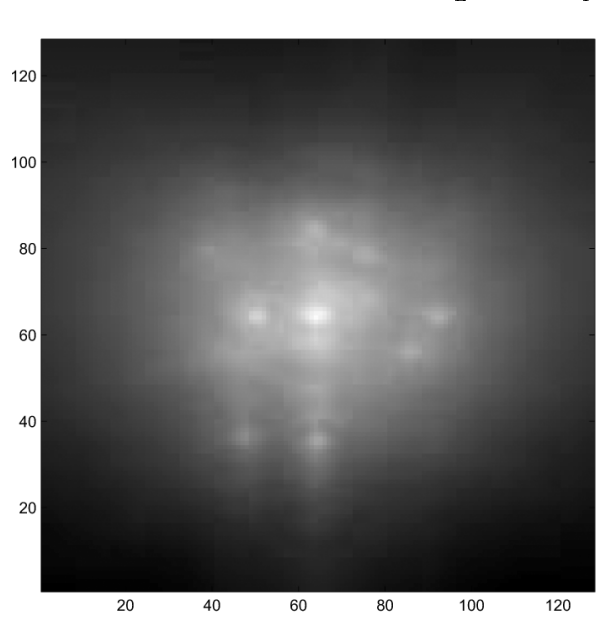

(a)

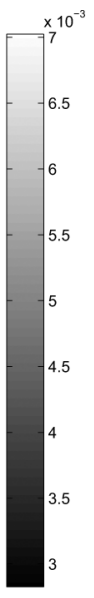

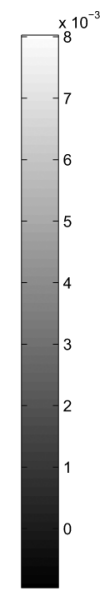

(b)

Fig. 23. Reconstructed images using (a) C-BP and (b) C-FBP for four noncooperative transmitters located at $\mathbf{y}_{0}, \mathbf{y}_{1}, \mathbf{y}_{2}$, and $\mathbf{y}_{3}$, and receivers traversing linear and parabolic flight trajectories $\gamma_{L}(s)$ and $\gamma_{P}(s)$ (see Fig. 17 for explicit formulae of $\gamma_{L}(s)$ and $\gamma_{P}(s)$ ).

\section{REFERENCES}

[1] N. Willis, Bistatic Radar. Norwood, MA: Artech House, 1991.

[2] IEEE Standard Radar Definitions, Radar Systems Panel of the IEEE Aerospace and Electronic Systems Society, IEEE Std. 686-1997, 1997.

[3] A. Horne and G. Yates, "Bistatic synthetic aperture radar," in Proc. IEEE RADAR, Oct. 2002 , pp. 6-10.

[4] H. Griffiths and N. Long, "Television-based bistatic radar," IEE Proc. Radar, Sonar, Navig., vol. 133, no. 7, pp. 649-657, 1986.
[5] P. Howland, D. Maksimiuk, and G. Reitsma, "Fm radio based bistatic radar," in IEE Proc. Radar, Sonar, Navig., Jun. 2005, vol. 152, pp. $107-115$.

[6] H. Griffiths and C. Baker, "Passive coherent location radar systems. Part 1: Performance prediction," IEE Proc. Radar, Sonar, Navig., vol. 152, no. 3, Jun. 2005.

[7] C. Baker, H. Griffiths, and I. Papoutsis, "Passive coherent location radar systems. Part 2: Waveform properties," IEE Proc. Radar, Sonar, Navig., vol. 152, no. 3, pp. 160-168, Jun. 2005. 
[8] D. Poullin, "Passive detection using digital broadcasters (DAB, DVB) with COFDM modulation," IEE Proc. Radar, Sonar, Navig., vol. 152, no. 3, pp. 143-152, Jun. 2005.

[9] D. Tan, H. Sun, Y. Lu, M. Lesturgie, and H. Chan, "Passive radar using global system for mobile communication signal: Theory, Implementation and measurements," IEE Proc. Radar, Sonar, Navig., vol. 152, no. 3, pp. 116-123, Jun. 2005.

[10] Roke Manor Research and FaberMaunsell Team to Drive Traffic Monitoring Technology Forward. [Online]. Available: http://www. roke.co.uk/press/46.php

[11] H. Griffiths, A. Garnett, C. Baker, and S. Keaveney, "Bistatic radar using satellite-borne illuminators of opportunity," in Proc. IEE Int. Conf. Radar, Oct. 1992, pp. 276-279.

[12] V. Koch and R. Westphal, "New approach to a multistatic passive radar sensor for air/space defense," IEEE Aerosp. Electron. Syst. Mag., vol. 10, pp. 24-32, Nov. 1995.

[13] M. Cherniakov, K. Kubik, and D. Nezlin, "Bistatic synthetic aperture radar with non-cooperative leos based transmitter," in Proc. IEEE Int. Geoscience and Remote Sensing Symp., Jul. 2000, vol. 2, pp. 861-862.

[14] M. Cherniakov, K. Kubik, and D. Nezlin, "Radar sensors based on communication low earth orbiting satellites microwave emission," in Proc. IEEE Int. Geoscience and Remote Sensing Symp., Jul. 2000, vol. 3, pp. 1007-1008.

[15] J. Homer, K. Kubik, B. Mojarrabi, I. Longstaff, E. Donskoi, and M. Cherniakov, "Passive bistatic radar sensing with leos based transmitters," presented at the IEEE International Geoscience and Remote Sensing Symp., 2002.

[16] M. Cherniakov, R. Saini, R. Zuo, and M. Antoniou, "Space surface bistatic sar with space-borne non-cooperative transmitters," in Proc. Eur. Radar Conf., Oct. 2005, pp. 25-28.

[17] L. Cutrona, , M. Skolnik, Ed., "Synthetic aperture radar," in Radar Handbook, 2nd ed. New York: McGraw-Hill, 1990, ch. ch. 21.

[18] X. He, M. Cherniakov, and T. Zeng, "Signal detectability in ss-bsar with gnss non-cooperative transmitter," IEE Proc. Radar, Sonar, Navig., vol. 152, no. 3, pp. 124-132, Jun. 2005.

[19] S. Nilsson, "Application of Fast Backprojection Techniques for Some Inverse Problems of Integral Geometry,” Ph.D. dissertation No. 499, Linköping Studies in Science and Technology, Linköping Univ., 1997.

[20] S. Norton and M. Linzer, "Backprojection reconstruction of random source distributions," J. Acoust. Soc. Amer., vol. 81, pp. 977-985, Apr. 1987.

[21] T.-K. Chan, Y. Kuga, and A. Ishimaru, "Experimental studies on circular SAR imaging in clutter using angular correlation function technique," IEEE Trans. Geosci. Remote Sens., vol. 37, no. 9, pp. 2192-2197, Sep. 1999.

[22] S. Norton and I. Won, “Time exposure acoustics," IEEE Trans. Geosci. Remote Sens., vol. 38, no. 5, pp. 1337-1343, May 2000.

[23] L. Borcea, G. Papanicolauou, and C. Tsogka, "Interferometric array imaging in clutter," Inv. Probl., vol. 21, pp. 1419-1460, 2005.

[24] S. Norton, B. Carr, and A. Witten, "Passive imaging of underground acoustic sources," J. Acoust. Soc. Amer., vol. 119, pp. 2840-2847, May 2006.

[25] Imaging of Complex Media With Acoustic and Seismic Waves., M. Fink, W. Kuperman, J.-P. Montagner, and A. Tourin, Eds. Berlin, Germany: Springer-Verlag, 2002.

[26] K. Wapenaar, D. Draganov, and J. Robertsson, Eds., "Seismic interferometry supplement," in Geophysics, Jul.-Aug. 2006, vol. 71, no. 4.

[27] E. Attia and B. Steinberg, "Self-cohering large antenna arrays using the spatial correlation properties of radar clutter," IEEE Trans. Antennas Propagat., vol. 37, no. 1, pp. 30-38, Jan. 1989.

[28] C. J. Nolan and M. Cheney, "Synthetic aperture inversion," Inv. Probl., vol. 18, pp. 221-236, 2002.

[29] C. Nolan and M. Cheney, "Synthetic aperture inversion for arbitrary flight paths and non-flat topography," IEEE Trans. Image Process., vol. 12, pp. 1035-1043, Dec. 2003.

[30] C. Yarman, B. Yazici, and M. Cheney, "Bistatic synthetic aperture radar imaging for arbitrary flight trajectories and non-flat topography," in Proc. IEEE Radar Conf., Apr. 2007, pp. 712-716.

[31] I.-Y. Son, T. Varslot, C. E. Yarman, A. Pezeshki, B. Yazici, and M. Cheney, "Radar detection using sparsely distributed apertures in urban environment," in Proc. SPIE Defense and Security Conf., Apr. 2007, p. $65671 Q$.
[32] R. J. Bonneau, "A rate distortion method for waveform design in RF target detection," in Proc. IEEE Aerospace Conf., 2006, pp. 1-12.

[33] M. Soumekh, "Wide-bandwidth continuous-wave monostatic/bistatic synthetic aperture radar imaging," in Proc. Inf. Conf. Image Processing, Oct. 1998, vol. 3, pp. 361-365.

[34] C. Yarman, B. Yazıc1, and M. Cheney, "Bistatic synthetic aperture radar imaging for arbitrary flight trajectories," IEEE Trans. Image Process., vol. 15, no. 1, pp. 84-93, Jan. 2006.

[35] H. Barrett and K. Myers, Foundations of Image Science. Hoboken, NJ: Wiley, 2004.

[36] F. Treves, Introduction to Pseudodifferential and Fourier Integral Operators. New York: Plenum, 1980, vol. I and II.

[37] E. Quinto, "Singularities of the X-ray transform and limited data tomography in $r^{2}$ and $r^{3}$," SIAM J. Math. Anal., vol. 24, pp. 1215-1225, 1993.

[38] B. Yazici, M. Cheney, and C. Yarman, "Synthetic-aperture inversion in the presence of noise and clutter," Inv. Probl., vol. 22, pp. 1705-1729, 2006.

[39] R. Courant and F. John, Introduction to Calculus and Analysis. New York: Springer, 2000, vol. $1 \& 2$

[40] F. Natterer and F. Wübbeling, Mathematical Methods in Image Reconstruction.. Philadelphia, PA: SIAM, 2001.

[41] N. Bleistein and R. Handelsman, Asymptotic Expansions of Integrals. New York: Dover, 1986.

[42] A. Grigis and J. Sjöstrand, Microlocal Analysis for Differential Operators: An Introduction, Ser. London Mathematical Society Lecture Note Series. Cambridge, U.K.: Cambridge Univ. Press, 1994, vol. 196.

[43] V. Guillemin and S. Sternberg, Geometric Asymptotics. Providence, RI: AMS, 1979.

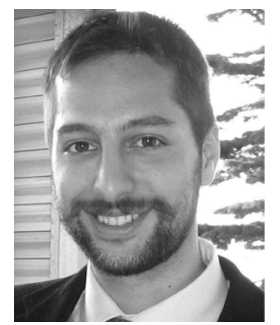

Can Evren Yarman (M'07) received the B.Sc. degree in mathematics from the Middle East Technical University, Ankara, Turkey, and the M.Sc. degree in biomedical science from Drexel University, Philadelphia, PA, and the M.Sc. degree in mathematics and the Ph.D. in electrical engineering from Rensselaer Polytechnic Institute (RPI), Troy, NY, in 2006.

He was a postdoctoral research associate at RPI from May 2006 to February 2007. Currently, he is a Research Scientist at the Houston Technology Center, WesternGeco-Schlumberger, Houston, TX. His main research interest is inverse problems in imaging.

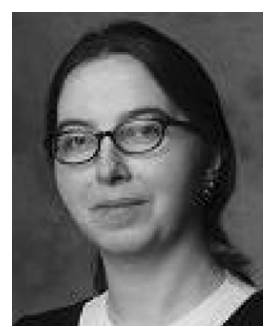

Birsen Yazıcı (SM'06) received the B.S. degrees in electrical engineering and mathematics from Bogazici University, Istanbul, Turkey, in 1988, and the M.S. and Ph.D. degrees in mathematics and electrical engineering from Purdue University, West Lafayette IN, in 1990 and 1994, respectively.

From September 1994 until 2000, she was a Research Engineer at the General Electric Company Global Research Center, Schenectady, NY. During her tenure in industry, she worked on radar, transportation, industrial, and medical imaging systems. In 2003, she joined Rensselaer Polytechnic Institute, Troy, NY, where she is currently an Associate Professor in the Department of Electrical, Computer, and Systems Engineering and in the Department of Biomedical Engineering. Her research interests span the areas of statistical signal processing, inverse problems in imaging, biomedical optics, and radar. She holds 11 U.S. patents.

Dr. Yazıcı is the recipient of the Rensselaer Polytechnic Institute 2007 School of Engineering Research Excellence Award. Her work on industrial systems received the 2 nd best paper award in 1997 given by IEEE TRANSACTIONS IN INDUSTRIAL APPLICATIONS. 